OPEN ACCESS

Edited by:

Ard Menzo Nijhof,

Freie Universität Berlin, Germany

Reviewed by:

Ben J. Mans,

Agricultural Research Council of South

Africa (ARC-SA), South Africa

Jianfeng Dai,

Soochow University, China

Shahid Karim,

University of Southern Mississippi,

United States

*Correspondence:

Jindriich Chmelar

chmelar@jcu.cz

Michail Kotsyfakis

mich_kotsyfakis@yahoo.com

${ }^{\dagger}$ These authors have contributed equally to this work.

Received: 25 February 2017 Accepted: 11 May 2017

Published: 29 May 2017

Citation:

Chmelar̆ J, Kotál J, Langhansová H and Kotsyfakis M (2017) Protease Inhibitors in Tick Saliva: The Role of

Serpins and Cystatins in

Tick-host-Pathogen Interaction.

Front. Cell. Infect. Microbiol. 7:216.

doi: 10.3389/fcimb.2017.00216

\section{Protease Inhibitors in Tick Saliva: The Role of Serpins and Cystatins in Tick-host-Pathogen Interaction}

\author{
Jindřich Chmelař ${ }^{1 * t}$, Jan Kotál ${ }^{1,2 \dagger}$, Helena Langhansová ${ }^{1,2}$ and Michail Kotsyfakis ${ }^{2 *}$ \\ ${ }^{1}$ Faculty of Science, University of South Bohemia in České Budějovice, České Budějovice, Czechia, ${ }^{2}$ Institute of Parasitology, \\ Biology Center, Czech Academy of Sciences, České Budějovice, Czechia
}

The publication of the first tick sialome (salivary gland transcriptome) heralded a new era of research of tick protease inhibitors, which represent important constituents of the proteins secreted via tick saliva into the host. Three major groups of protease inhibitors are secreted into saliva: Kunitz inhibitors, serpins, and cystatins. Kunitz inhibitors are anti-hemostatic agents and tens of proteins with one or more Kunitz domains are known to block host coagulation and/or platelet aggregation. Serpins and cystatins are also anti-hemostatic effectors, but intriguingly, from the translational perspective, also act as pluripotent modulators of the host immune system. Here we focus especially on this latter aspect of protease inhibition by ticks and describe the current knowledge and data on secreted salivary serpins and cystatins and their role in tick-host-pathogen interaction triad. We also discuss the potential therapeutic use of tick protease inhibitors.

Keywords: tick-host interaction, immunomodulation, protease inhibitors, serpins, cystatins

\section{SERPINS AND CYSTATINS AS HOMEOSTATIC REGULATORS}

Proteases (also proteinases or peptidases) are ubiquitous enzymes that cleave proteins to smaller peptides and amino acids. Proteases participate in a range of physiological processes including extracellular digestion, protein degradation, and tissue development (Rawlings and Salvesen, 2013). Relevant to this review, however, is the fact that many proteases, in particular highly substrate-specific endopeptidases, mediate defense and homeostatic processes in both vertebrates and invertebrates. Proteolytic pathways rely on the precise and tightly regulated activation and inhibition of these endopeptidases. As a result of this evolutionary need, many crucial pathophysiological processes are regulated via proteolytic cascades, with notable examples being coagulation of plasma (or haemolymph in arthropods), bacterial wall perforation with complement, or melanization in arthropods (Amara et al., 2008; Tang, 2009; Gulley et al., 2013). Each step involves proteolytic activation of another downstream protease, and all proteases in such cascades usually have their own endogenous inhibitors that balance the system. The role of arthropod protease inhibitors in the defense is supported by the fact that the expression of serpins and cystatins in Ixodes scapularis nymphs was attenuated upon infection with Anaplasma phagocytophilum, as seen in the transcriptomic data (Ayllon et al., 2015). On the other hand, the expression of protease inhibitors in salivary glands and midguts of adult females differed among individual inhibitors, i.e., some cystatins and serpins were upregulated upon the infection and vice versa (Ayllon et al., 2015). Similar data were collected from Ixodes ricinus infected with Bartonella henselae (Liu et al., 2014). Therefore, precise involvement of every individual inhibitor in tick infection would have to be evaluated experimentally. 
Other intracellular and extracellular processes, such as cytokine activation, phagocytosis, intracellular signaling, and antigen processing, are also dependent on proteolysis (Muller et al., 2012). Serpins and cystatins are the two main superfamilies of endogenous serine and cysteine protease inhibitors involved in the regulation of these processes. It is therefore unsurprising that both groups of inhibitors are well represented in parasites and are important in their interactions with hosts (Schwarz et al., 2012; Meekins et al., 2017). In order to obtain a blood meal, ticks secrete hundreds of different pharmacoactive molecules into the host via their saliva. These molecules have anti-hemostatic, anti-inflammatory, anti-complement and immunomodulatory properties and their function is to overcome or evade host defense mechanisms including immune response (Brossard and Wikel, 2004; Chmelar et al., 2012). Moreover, tick saliva and also several salivary compounds were found to facilitate and enhance the establishment of tick-borne pathogens in the host (Anguita et al., 2002; Pal et al., 2004; Kazimirova and Stibraniova, 2013; Wikel, 2013). Inhibitors of proteases represent the most prominent protein families in tick salivary secretion that are responsible for alteration of many different host defense pathways.

\section{SERINE PROTEASE INHIBITORS IN TICKS}

Four groups of serine protease inhibitors have been described in ticks: Kunitz domain inhibitors, Kazal domain inhibitors, trypsin inhibitor-like cysteine rich domain (TIL) inhibitors, and serpins. Inhibitors with 1-7 Kunitz domains mostly act as anti-hemostatic proteins and form a large multigenic family of secreted salivary proteins in ticks that have probably played a crucial role in the development of tick hematophagy (Corral-Rodriguez et al., 2009; Dai et al., 2012; Schwarz et al., 2014). Moreover, single Kunitzdomain inhibitors in other organisms are involved in ion channel blockade and may play a similar role in ticks (Frazao et al., 2012; Valdes and Moal, 2014). Kazal domain inhibitors are described in hematophagous insects such as mosquitoes and triatomine bugs (Rimphanitchayakit and Tassanakajon, 2010), but they are only rarely reported in ticks, in which their function is still unknown (Zhou et al., 2006a; Mulenga et al., 2007a, 2008). TIL-domain inhibitors represent an interesting group of small inhibitors with a conserved 5-disulphide bridge structure that were first reported in Apis melifera (Bania et al., 1999) and have also been detected in ticks (Fogaca et al., 2006; Sasaki et al., 2008). The sequences of over 80 TIL-domain inhibitors have been found in arthropod genomes (Zeng et al., 2014), and the unique features of TILdomain proteins make them an excellent model for designing novel serine protease inhibitors and antimicrobial peptides ( $\mathrm{Li}$ et al., 2007).

\section{Serpins}

Serpins form the largest superfamily of protease inhibitors, and they are ubiquitously distributed in nature including viruses and prokaryotes. With over 1,500 members, serpins are the most studied protease inhibitors (Law et al., 2006), also helped by their unique and highly intriguing mechanism of inhibition (Whisstock et al., 2010) and the evolutionary changes that turned inhibitory serpins into non-inhibitory proteins with completely different functions (Law et al., 2006; Silverman et al., 2010). For example, there are 29 inhibitory and seven non-inhibitory serpins in humans and 60 functional serpin genes in mice (Heit et al., 2013). Angiotensinogen is a non-inhibitory serpin that is proteolytically activated by renin into several oligopeptides (angiotensins) that regulate vasoconstriction and blood pressure (Lu et al., 2016). Cortisol and thyroxine-binding proteins (human SERPINA6 and SERPINA7) are also notable serpins that act as major transport proteins for glucocorticoids and progesterone (Carrell and Read, 2016). Inhibitory serpins have very diverse functions depending on their specificity, but their importance is highlighted by the serpinopathies-diseases caused by serpin dysfunction or deficiency (Belorgey et al., 2007). Emphysema, cirrhosis, angioedema, hypertension, and even familial dementia are caused at least in part by serpin dysfunction (Kim et al., 1995; Davis et al., 1999; Ekeowa et al., 2009; Huntington and Li, 2009; Lomas et al., 2016).

Arthropod serpins have mostly immunological and hemostatic functions. Serpins have been shown to regulate haemolymph coagulation, are involved in phenoloxidase system activation in insects, and regulate an immune toll pathway in haemolymph (Kanost, 1999; Gulley et al., 2013; Meekins et al., 2017). Furthermore, in bloodfeeding arthropods, serpins can act as modulators of host hemostasis and/or immune responses. Indeed, several insect serpins act as anti-coagulants, anti-complement proteins and immunosuppressors (Stark and James, 1995, 1998; Colinet et al., 2009; Calvo et al., 2011; Ooi et al., 2015). Serpins are abundant in ticks, and one of their functions is to modulate host immune system. Recent advances in this area have been facilitated by the publication of I. scapularis genome (Gulia-Nuss et al., 2016) and several next-generation sequencing transcriptome studies that added tens of unique sequences from different tick species to already existing and long list of tick serpins. In 2009, Mulenga and colleagues found 45 serpins in the genome of I. scapularis (Mulenga et al., 2009). Two years earlier, the same group described 17 serpins (Lospins) in Amblyomma americanum (Mulenga et al., 2007b). This number was, however, substantially broadened by the combination of several approaches up to approximately 120 serpins (Karim and Ribeiro, 2015; Porter et al., 2015, 2017). In the work of Porter and colleagues (Porter et al., 2015), the authors compare homologous serpins across tick species, showing both conserved and species-specific inhibitors. The conservation seems to be higher in serpins with basic or polar uncharged amino acid residues at P1 site (Porter et al., 2015). Other 32 serpin transcripts from the Amblyomma genus were found in Amblyomma maculatum (Karim et al., 2011) and 50 in Amblyomma sculptum (Moreira et al., 2017). Two groups described 18 and 22 serpins in $R$. microplus, respectively (Tirloni et al., 2014b; Rodriguez-Valle et al., 2015) and at least 36 serpins were found in several published trancriptomes from I. ricinus (our own unpublished data based on the analysis of transcriptomes) (Schwarz et al., 2013; Kotsyfakis et al., 2015a,b; Perner et al., 2016). Another recent publication described 10 different serpin transcripts in the sialotranscriptome of the tick Hyalomma excavatum (Ribeiro et al., 2017). Despite 
high number of identified transcripts, only small portion was characterized functionally.

\section{Tick Serpins with Known Function}

To date, almost 20 tick serpins from different tick species have been functionally validated by in vitro assays, in vivo experimental models, vaccination and by RNA interference (RNAi) experiments (Table 1). These are detailed below.

\section{AamS6 (A. americanum)}

Only two serpins (AamS6 and AAS19) have been characterized thus far in A. americanum, despite the overall high number of serpins identified in this tick (Porter et al., 2015). A. americanum serpin 6 (AamS6) is upregulated during first 3 days of feeding and is likely to be injected into the host during feeding; however, RNAi did not affect tick feeding ability (Chalaire et al., 2011). Recombinant AamS6 inhibited the serine proteases trypsin, chymotrypsin, elastase, and chymase and the cysteine protease papain in a dose-dependent manner (Chalaire et al., 2011). AamS6 also reduced platelet aggregation and delayed plasma clotting time, suggesting that this serpin facilitates blood feeding by ticks (Mulenga et al., 2013). The complement activation pathway, however, was not affected (Mulenga et al., 2013).

\section{AAS19 (A. americanum)}

AAS19 is an anti-coagulant that was shown to inhibit five of the eight serine protease blood clotting factors. AAS19 inhibited thrombin-but not ADP-and cathepsin G-activated platelet aggregation and delayed clotting in re-calcification and thrombin time assays (Kim et al., 2015). AAS19 RNAi halved the blood intake and resulted in morphological deformation of ticks (Kim et al., 2016). In rabbits, immunized with AAS19, tick feeding was faster, but smaller blood volumes were ingested, and tick ability to lay eggs was impaired (Kim et al., 2016).

\section{HLS-1 and 2 (Haemaphysalis longicornis)}

Sugino and colleagues isolated a serpin from $H$. longicornis in 2003 (HLS1) (Sugino et al., 2003). Recombinant HLS1 displayed anticoagulant activity, and nymph and adult tick feeding on immunized rabbits resulted in 43.9 and $11.2 \%$ tick mortality, respectively. Antibodies raised against tick saliva did not react with recombinant HSL1, suggesting that the serpin was not secreted (Sugino et al., 2003). Moreover, HLS1 expression was detected in the midgut rather than the salivary glands, and HLS1 was therefore considered a concealed antigen, similar to the first commercially used anti-tick vaccine based on the Bm86 tick protein (Willadsen et al., 1995). HLS1 does not contain a signal peptide. Therefore, it is likely that HLS1 is not a secreted protein playing an immunomodulatory or anti-hemostatic role in the host during tick feeding.

A second serpin from $H$. longicornis (HLS2) possesses a signal sequence and seems to be secreted by hemocytes into the haemolymph but not by the salivary glands or midgut (Imamura et al., 2005). HLS2 prolonged the coagulation time in a dosedependent manner (Imamura et al., 2005), and rabbit vaccination with HLS2 resulted in greater immunization than with HLS1 and almost 50\% mortality of feeding nymphs and adults (Imamura et al., 2005). This might be explained by better accessibility and inactivation of extracellular HLS2 in the haemolymph by antibodies from the ingested blood of immunized animals.

\section{Ipis-1 (Ixodes persulcatus)}

To date, Ipis-1 is the only characterized salivary serpin from tick I. persulcatus (Toyomane et al., 2016). Ipis-1 transcripts were detected only in salivary glands of ticks at same level throughout all phases of feeding. It significantly reduced IFN- $\gamma$ production and the proliferation of bovine PBMC cells after ConA stimulation. Authors suggest that Ipis-1 could inhibit T cells function by direct interaction with this cell population (Toyomane et al., 2016).

\section{Iris (I. ricinus)}

The first tick serpin to be described that had an effect on host defense mechanisms was named Iris (Ixodes ricinus immunosuppressor) (Leboulle et al., 2002a,b). Iris displayed several notable and important features. First, Iris was noted to inhibit $\mathrm{T}$ cell and splenocyte proliferation and altered peripheral blood mononuclear cell (PBMC)-derived cytokine levels (Leboulle et al., 2002a). Second, Iris showed antihemostatic properties including suppression of coagulation and fibrinolysis (Prevot et al., 2006). Finally, Iris was shown to bind to monocytes/macrophages and suppress the secretion of TNF (Prevot et al., 2009). Interestingly, these activities were independent on the protease inhibitory function of Iris. Of note, Iris, together with HLS1 and several other proteins, belongs to a group of serpins in Ixodes spp. that have methionine and cysteine in their reactive center loop (RCL) and lack a signaling peptide, suggesting intracellular rather than extracellular function. However, Iris has been detected in tick saliva using a polyclonal serum raised against recombinant protein (Leboulle et al., 2002a; Prevot et al., 2007), and vaccination of rabbits with recombinant Iris increased the mortality of feeding ticks and lowered weight after engorgement (Prevot et al., 2007). This contradictory observation might be explained by cross-reactivity with another secreted serpin or by the action of another, non-classical secretory mechanism (Nickel, 2003). Nevertheless, Iris represents a pleiotropic protein that affects multiple processes simultaneously via independent mechanisms.

\section{IRS-2 (I. ricinus)}

IRS-2 (Ixodes ricinus serpin-2) was the second serpin to be characterized in I. ricinus. IRS-2 has tryptophan in its P1 site, confirmed by its resolved crystal structure (Kovarova et al., 2010; Chmelar et al., 2011). IRS-2 displayed inhibitory specificity against mast cell chymase and cathepsin G, two proteases involved in inflammatory responses (Chmelar et al., 2011), with its anti-inflammatory function evidenced by in vivo paw edema experiments, in which IRS-2 significantly decreased paw swelling and neutrophil recruitment in treated animals (Chmelar et al., 2011). Moreover, IRS-2 inhibited the production of proinflammatory cytokine IL-6 in dendritic cells (DC) and impaired IL-6-dependent JAK/STAT3 signaling in T-helper (Th) cells, inhibiting the maturation of proinflammatory Th17 lymphocytes (Palenikova et al., 2015). IRS-2 also inhibited 


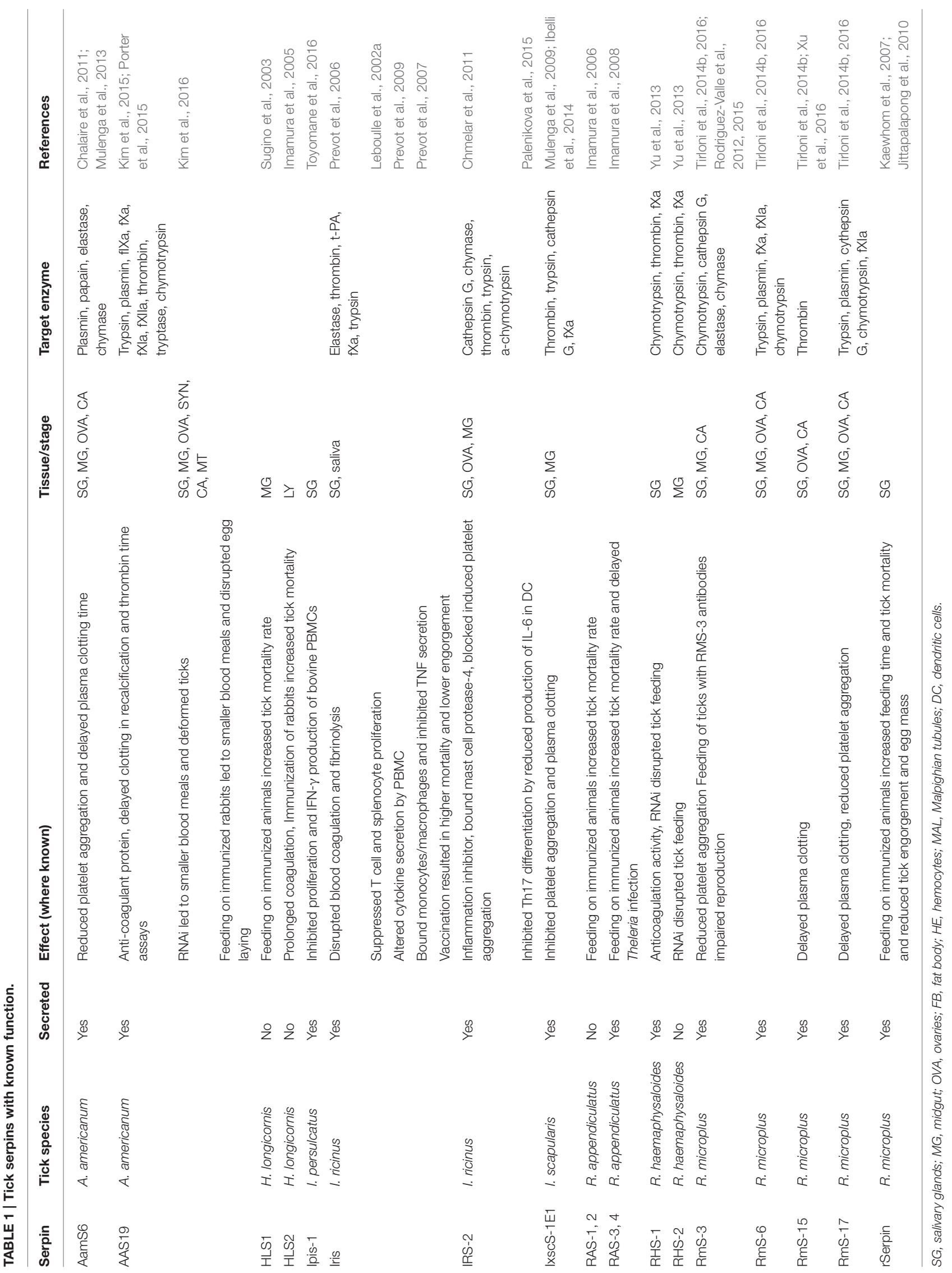


platelet aggregation induced by cathepsin $G$ but not other inducers such as collagen or arachidonic acid derivatives (Chmelar et al., 2011).

\section{IxscS-1E1 (I. scapularis)}

A blood meal-induced salivary serpin IxscS-1E1 from $I$. scapularis has been shown to trap thrombin and trypsin in SDS- and heat-stable complexes, reduce their activity and inhibit the activities of cathepsin $\mathrm{G}$ and factor $\mathrm{Xa}$, although protease/inhibitor complexes were not detected (Ibelli et al., 2014). Furthermore, IxscS-1E1 inhibited adenosine diphosphateand thrombin-activated platelet aggregation and delayed plasma clotting time, suggesting an anti-hemostatic role (Ibelli et al., 2014). IxscS-1E1 had no effect on the classical complement activation pathway (Ibelli et al., 2014).

\section{RAS-1, 2, 3, 4 (Rhipicephalus appendiculatus)}

Four serpin cDNAs, two putatively secreted (RAS-3 and RAS4) and two putatively intracellular (RAS-1 and RAS-2), were identified in and isolated from the salivary glands of $R$. appendiculatus (Mulenga et al., 2003). Although RAS-1 and RAS-2 are expressed in the salivary glands, antibodies against them were not found at the bite site as determined by the reactivity of anti-tick saliva sera to recombinant RAS-1 and RAS2 (Imamura et al., 2006). This finding is, however, consistent with their predicted intracellular location (Imamura et al., 2006). Vaccination of cattle with a RAS-1/RAS-2 cocktail resulted in a $61.4 \%$ reduction in nymph engorgement rate and a 28 and $43 \%$ increase in mortality rate in female and male adult ticks, respectively (Imamura et al., 2006). Similar results were obtained when cattle were vaccinated with a mixture of two secreted serpins RAS-3 and RAS-4 and a $36-\mathrm{kDa}$ immunodominant cement protein RIM36 (Imamura et al., 2008): immunization resulted in $40 \%$ mortality rate for $R$. appendiculatus ticks and almost 50\% for Theileria parva-infected female ticks (Imamura et al., 2008). However, no significant protective effect against infection with $T$. parva was observed in spite of a 1-2 day delay in the detection of pathogens in the host peripheral blood after immunization (Imamura et al., 2008).

\section{RHS-1 and 2 (Rhipicephalus haemaphysaloides)}

Two serpins (RHS-1 and RHS-2) have been identified and characterized from R. haemaphysaloides (Yu et al., 2013), both of which were expressed in the salivary glands and midguts of ticks fed for 4 days. Both inhibited chymotrypsin, and RHS-1 also inhibited thrombin (Yu et al., 2013). Consistent with their inhibitory activity, only RHS-1 exhibited anticoagulation activity based on the activated partial thrombin time assay (Yu et al., 2013). Only RHS- 1 seems to be secreted into the saliva and the host, as only RHS-1 was detected by serum from rabbits that were exposed to ticks and only RHS-1 possesses a signal peptide sequence (Yu et al., 2013). Nevertheless, RNAi of both serpins negatively affected the attachment rate after $24 \mathrm{~h}$ and decreased the engorgement rate (Yu et al., 2013).

\section{RmS-3, 6, 15, 17 (R. microplus)}

Serpin RmS-3 from $R$. microplus displayed anti-elastase and anti-chymotrypsin inhibitory activities (Rodriguez-Valle et al.,
2015). Tirloni and colleagues subsequently confirmed this specificity (albeit with much lower inhibitory activity), tested more proteases, and found the highest inhibitory activity against chymase and cathepsin G (Tirloni et al., 2016). RmS3 is likely to be secreted into the saliva and the host as evidenced by differential antibody responses of tick-resistant and tick-susceptible cattle (Rodriguez-Valle et al., 2012). RmS-3 is expressed in nymphs and in the salivary glands of adult ticks, data on RmS-3 transcription in ovaries differ between the two studies (Tirloni et al., 2014b; Rodriguez-Valle et al., 2015). Capillary feeding of ticks with a RmS-3 antibody reduced tick reproductive capacity (Rodriguez-Valle et al., 2012, 2015).

In addition to $\mathrm{RmS}-3$, three other recombinant $R$. microplus serpins were produced for enzymatic and functional characterization (Tirloni et al., 2014a,b; Xu et al., 2016). RmS-6 inhibited factor Xa, factor XIa and plasmin, suggesting an anticoagulant function, while RmS-17 showed weaker inhibitory activity against chymotrypsin, cathepsin G, trypsin, and plasmin (Tirloni et al., 2016). Both RmS-3 and RmS-17 inhibited cathepsin G-induced platelet aggregation. Interestingly, RmS-3, 6 , and -17 from $R$. microplus were recognized by antibodies raised by the saliva of $A$. americanum, I. scapularis, and Rhipicephalus sanguineus, suggesting a potential use for these proteins as an universal tick vaccine (Tirloni et al., 2016) but also highlighting the pitfall of false-positive detection of serpins in tick saliva. RmS-15 was identified as a thrombin inhibitor and, together with RmS-17, delayed plasma clotting in a re-calcification time assay (Tirloni et al., 2016; Xu et al., 2016). Moreover, RmS-15 is an immunogen, as the infestation of cattle with $R$. microplus resulted in increased anti-RmS-15 IgG titers (Xu et al., 2016).

\section{rSerpin (R. microplus)}

Rabbits immunized with putatively secreted serpin (rSerpin) from $R$. microplus (Kaewhom et al., 2007) led to extended feeding time, an $83 \%$ reduction in adult engorgement, $67 \%$ mortality of engorged females and a $34 \%$ reduction in egg mass weight (Jittapalapong et al., 2010).

\section{Cystatins}

Cystatins form a superfamily of tight-binding reversible inhibitors of papain-like cysteine proteases and legumains and, similar to serpins, they are present in all organisms including prokaryotes (Kordis and Turk, 2009). Cystatins regulate many physiological processes including immunity-related mechanisms such as antigen presentation, phagocytosis, and cytokine expression (Zavasnik-Bergant, 2008). There are four cystatin subgroups: type 1 (stefins), type 2, type 3 (kininogens), and type 4 cystatins (fetuins) (Rawlings and Barrett, 1990). Cystatins' target proteases are usually lysosomal cathepsins involved in protein degradation, but they also target those involved in degradation of antigens presented via MHCII to lymphocytes or in the activation of caspase 1 and thus inflammasome regulation (Jin and Flavell, 2010; Turk et al., 2012).

\section{Cystatins with Known Function}

Similarly to serpins, there are around 20 tick cystatins described in the literature and only type 1 and type 2 cystatins have thus 
far been reported in ticks. While stefins lack a secretory signal and are most likely involved in the intracellular digestion of hemoglobin or in developmental processes, type 2 cystatins are secreted and expressed in both the midgut and salivary glands (Schwarz et al., 2012). Tick cystatins either regulate hemoglobin digestion, which is driven by cathepsins (Horn et al., 2009), or they can be secreted as immunomodulators into the host with saliva. The majority (84\%) of tick cystatin transcripts that are conserved across tick species, belong to the extracellular group, suggesting predominantly immunomodulatory role (Ibelli et al., 2013) Tick cystatins with experimentally validated functions are listed in Table 2 and detailed below.

\section{Bmcystatin (R. microplus)}

Bmcystatin from $R$. microplus is specifically expressed in the salivary glands, ovaries, and fat bodies. Bmcystatin did not inhibit papain but inhibited human cathepsin L and tick vitellindegrading cysteine endopeptidase (VDTCE), suggesting a role in regulating tick embryogenesis (Lima et al., 2006).

\section{BrBmcys $2 a, b, c, d, e,($ R. microplus)}

In addition to Bmcystatin, another five cystatins (BrBmcys2a, b, c, d, e) were identified in the cattle tick $R$. microplus. Their expression differs among various developmental stages and tissues, but since their presence has only been assessed by immunodetection methods, cross reactivity between antibodies is possible and has indeed been reported (Imamura et al., 2013). This study also examined the inhibitory specificity of two cystatins: while BrBmcys $2 \mathrm{~b}$ targeted cathepsins B, C, and L, BrBmcys2c only inhibited cathepsins C and L (Parizi et al., 2015). Antibodies raised against recombinant proteins detected BrBmcys $2 b$ in all tick tissues, while anti-BrBmcys $2 c$ serum only recognized the protein in the gut from partially engorged females and in the ovaries, salivary glands, and fat bodies from fully engorged females (Parizi et al., 2015). The expression patterns suggest rather homeostatic function of these cystatins in ticks than immunomodulatory activity in the host (Imamura et al., 2013).

\section{Cystatin (A. americanum)}

One cystatin was detected in the salivary glands and midguts of unfed and partially fed A. americanum ticks (Karim et al., 2005). RNAi of this cystatin led to a 90 and 50\% reduction in transcript abundance in the early and late phases of feeding, respectively. RNAi knockdown decreased tick body weight, killed ticks during feeding, and disrupted feeding to full engorgement. Rabbits preexposed to dsRNA-injected ticks were re-exposed to naïve ticks, which led to detachment of $34 \%$ ticks after 1 day and over $50 \%$ mortality of attached ticks (Karim et al., 2005). No such effect was observed in the control group, in which rabbits were pre-exposed to normal ticks. Such a strong immune response indicates an important immunomodulatory function for silenced cystatin that impairs responses to salivary antigens and leads to an overall less intense immune reaction (Karim et al., 2005).

\section{HISC-1 (H. longicornis)}

HISC- 1 is a type 2 cystatin detected in H. longicornis (Yamaji et al., 2009b). It is found mainly in the acinar cells of the tick salivary glands and is therefore likely to be secreted into the host. The number of transcripts was found to be approximately 5 -fold higher in the salivary glands than in the midgut, with strong upregulation in early phase of blood feeding and with a pattern suggestive of importance in the feeding process. HISC-1 inhibited cathepsins L and papain but not cathepsin B (Yamaji et al., 2009b).

\section{Hlcyst-1, 2 and 3 (H. longicornis)}

While Hlcyst-1 is a type 1 intracellular cystatin with specificity against papain and cathepsin L (Zhou et al., 2009), Hlcyst-2 and Hlcyst- 3 are secreted type 2 cystatins (Zhou et al., 2006b, 2010). Hlcyst-2 has been shown to inhibit cathepsin L and cathepsin B, with transcripts found mainly in the midgut and hemocytes of all tick developmental stages. Expression increased with tick development and was induced by blood feeding (Zhou et al., 2006b). Moreover, Hlcyst-2 expression was induced by injecting ticks with LPS or Babesia gibsoni, suggesting a role in tick immunity. In vitro cultivation of $B$. gibsoni in the presence of Hlcyst-2 significantly inhibited pathogen growth (Zhou et al., 2006b). Hlcyst-1 and Hlcyst-2 also inhibited cysteine protease HlCPL-A with hemoglobinase activity, isolated from $H$. longicornis, which can act as natural target of these cystatins, suggesting an involvement of both the protease and its inhibitors in blood digestion (Yamaji et al., 2009a). Hlcyst-3 inhibited papain and cathepsin L, and its expression was detected preferentially in the midgut (Zhou et al., 2010).

\section{JpIocys2 (Ixodes ovatum)}

JpIocys 2 was isolated from $I$. ovatum and was shown to modulate the enzymatic activity of cathepsins B, C, and L with cathepsin L as the preferred target (Parizi et al., 2015). Similar to BrBmcys2b and BrBmcys $2 \mathrm{c}$, JpIocys 2 is considered to be involved in tick homeostasis and egg development.

\section{JpIpcys $2 a, b, c$ (I. persulcatus)}

Three novel cystatins from I. persulcatus, JpIpcys $2 \mathrm{a}, \mathrm{b}$, and $\mathrm{c}$, have recently been described in terms of sequence and structural analysis and expression profile (Rangel et al., 2017). All three possess a signal peptide and two disulfide bridges in their mature form. Although varying in their tertiary structure, all three $I$. persulcatus cystatins should bind human cathepsin L and papain, based on in silico analyses. Transcripts of all three cystatins were detected in almost all tissues (salivary glands, midgut, carcass) and stages (larvae, nymphs, adults) of tick development. The only exception was absence of JpIpcys2c transcripts in unfed larvae. Furthermore, vaccination of hamsters with a structurally similar BrBmcys2c cystatin from $R$. microplus did not show any crossreactivity and did not lead to impaired $I$. persulcatus feeding or reproduction (Rangel et al., 2017).

\section{Om-cystatin 1 and 2 (Ornithodoros moubata)}

Om-cystatin 1 and 2 were described in a soft tick O. moubata (Grunclova et al., 2006). While Om-cystatin 1 transcripts were found only in the midguts of unfed ticks, Om-cystatin 2 mRNA was present in all tissues. Transcript levels were rapidly suppressed after tick feeding. Both possessed inhibitory activity against cathepsins B, C, and $\mathrm{H}$ and papain (Grunclova et al., 


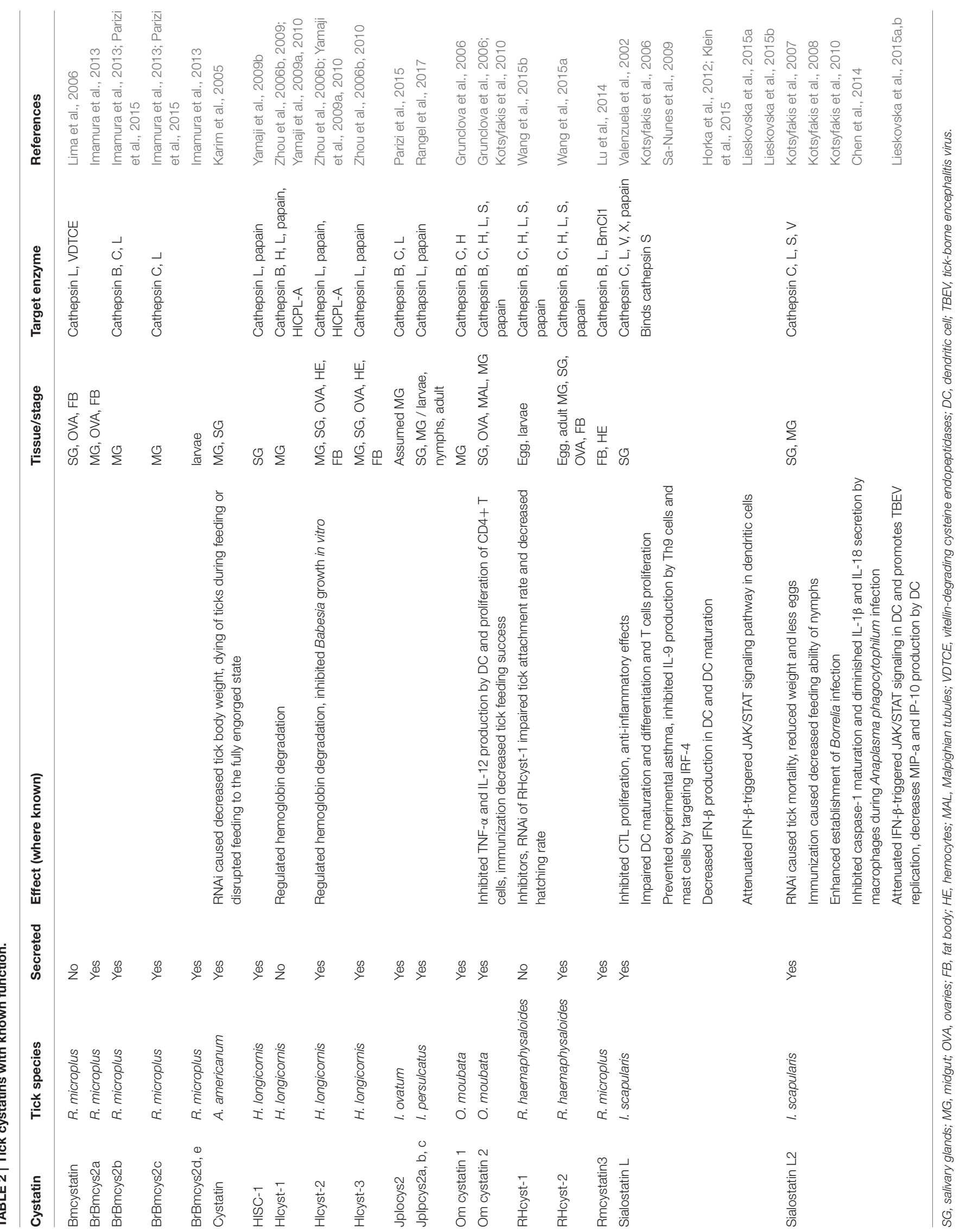


2006). Om-cystatin 2 was further functionally and structurally characterized under the name OmC2 (Salát et al., 2010). OmC2 inhibited the secretion of pro-inflammatory cytokines TNF and IL-12 by DC after LPS stimulation and reduced antigen-specific $\mathrm{CD} 4^{+} \mathrm{T}$ cell proliferation induced by DC (Salát et al., 2010). Exposing OmC2 immunized mice to O. moubata nymphs reduced feeding ability and increased mortality during nymphal development to the next stage. Interestingly, nymphs mortality was positively correlated with higher titers of antiOmC2 antibodies in the serum (Salát et al., 2010).

\section{RHcyst-1 and RHcyst-2 (R. haemaphysaloides)}

Two cystatins have been described in $R$. haemaphysaloides, RHcyst-1 and RHcyst-2. RHcyst-1 is an intracellular type 1 cystatin that inhibited cathepsins $\mathrm{L}, \mathrm{B}, \mathrm{C}, \mathrm{H}$, and $\mathrm{S}$ and papain, with strongest affinity to cathepsin S (Wang et al., 2015b). RHcyst-1 was expressed at all developmental stages but was most abundant in tick eggs, and its expression decreased throughout the development. RNAi of RHcyst-1 reduced the attachment rate of adult ticks and decreased hatching rate (Wang et al., 2015b). RHcyst-2 is a secreted type 2 cystatin that inhibited the same cathepsins as RHcyst-1 (Wang et al., 2015a) and was again present at all developmental stages with highest expression in eggs. However, RHcyst-2 expression increased during blood feeding, and RHcyst-2 was secreted to the host during tick feeding according to immunodetection methods (Wang et al., 2015a).

\section{Rmcystatin3 (R. microplus)}

Rmcystatin3 inhibited cathepsins L and B and Boophilus

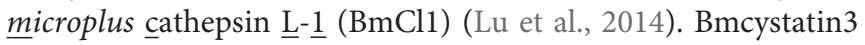
transcripts were found in tick hemocytes, fat bodies, and salivary glands, but protein was only detected in hemocytes and the fat bodies by western blotting. Infection of ticks with $E$. coli significantly downregulated Bmcystatin3 expression ( $\mathrm{Lu}$ et al., 2014) but increased efficacy of pathogen clearance, suggesting that Rmcystatin3 may be a negative regulator of tick immune responses, probably by regulating cysteine proteases responsible for the production of antimicrobial effectors in hemocytes ( $\mathrm{Lu}$ et al., 2014).

\section{Sialostatin L (I. scapularis)}

One of the best studied tick cystatins is sialostatin L, a type 2 cystatin detected in I. scapularis. Sialostatin L has preferential specificity for cathepsin L; however, cathepsins V, C, X, S, and papain were also inhibited in enzymatic assays (Kotsyfakis et al., 2006). In the same study, sialostatin $L$ inhibited the proliferation of the cytotoxic T lymphocyte cell line CTLL-2, suggesting its effect on adaptive immunity. Moreover, the anti-inflammatory activity of sialostatin $\mathrm{L}$ was confirmed in a mouse model of carrageenan-induced paw edema, in which sialostatin $L$ reduced edema and neutrophil myeloperoxidase activity (Kotsyfakis et al., 2006).

Sialostatin L has been shown to inhibit IL-2 and IL-9 production by Th9 lymphocytes (Horka et al., 2012). IL-9 production by Th cells is IL-2 dependent (Schmitt et al., 1994), but the addition of exogenous IL-2 did not rescue IL-9 synthesis, suggesting that mechanisms other than IL-2 reduction may be involved in IL-9 inhibition (Horka et al., 2012). Nevertheless, the impairment of Th9 cells by sialostatin $\mathrm{L}$ abrogated the eosinophilia and airway hyperresponsiveness of mice challenged with OVA antigen (Horka et al., 2012). The inhibition of IL9 production together with reduced expression of IL-1 $\beta$ and IRF4 (interferon regulating factor 4) was also observed in mast cells, with IL-9 production rescued by the application of exogenous IL-1 $\beta$ (Klein et al., 2015). The inhibition of IL-9 was IRF4 or IL-1 $\beta$ dependent, as proven by the fact that IRF4deficient or IL-1 receptor-deficient mast cells failed to produce IL-9. The transcription factor IRF4 binds to IL-1 $\beta$ and IL-9 promoters, implying that sialostatin L inhibits IL-9 production via its effect on IRF4 (Klein et al., 2015). Furthermore, mice with IRF4 knockdown in mast cells or mice administered with sialostatin $\mathrm{L}$ showed a strong reduction in eosinophilia and airway hyperresponsiveness, important symptoms of asthma. Conversely, sialostatin L did not affect mast cell degranulation or IL-6 expression (Klein et al., 2015).

Sialostatin L inhibits cathepsin S, resulting in reduced antigenspecific $\mathrm{CD}^{+} \mathrm{T}$ cell proliferation in vitro and in vivo; sialostatin $\mathrm{L}$ treatment during OVA immunization impaired early $\mathrm{T}$ cell expansion of splenocytes in OT-II mice and late recall immune responses by impairing the proliferation of lymph node cells (Sa-Nunes et al., 2009). Sialostatin L also potently prevented symptoms of experimental autoimmune encephalomyelitis in mice accompanied by impaired IFN- $\gamma$ and IL-17 production and specific T cell proliferation (Sa-Nunes et al., 2009).

In addition to modulating $\mathrm{T}$ cells, sialostatin $\mathrm{L}$ inhibited $\mathrm{DC}$ maturation and reduced the production of IL-12 and TNF by DC (Sa-Nunes et al., 2009). These effects on DC can also be attributed to anti-cathepsin $\mathrm{S}$ activity, as cathepsin $\mathrm{S}$ plays a role in an invariant chain processing (Pierre and Mellman, 1998) and its inhibition thus leads to poor antigen presentation by DC (Sa-Nunes et al., 2009). Similar to another I. scapularis cystatin Sialostatin L2 (Lieskovska et al., 2015b), sialostatin L attenuated IFN- $\beta$-triggered JAK/STAT signaling in DC (Lieskovska et al., 2015a). However, unlike Sialostatin L2, it did not suppress expression of the IP-10 chemokine or IRF-7, suggesting that these two cystatins can produce the same phenotype by impairing different pathways in the same cell (Chmelar et al., 2016). It also decreased IFN- $\beta$ production in DC activated by either Borrelia or TLR-7 ligand (Lieskovska et al., 2015a).

\section{Sialostatin L2 (I. scapularis)}

Sialostatin L2 is an I. scapularis cystatin similar in sequence to sialostatin $\mathrm{L}$ but with different anti-protease potency, antigenicity, and expression pattern. Unlike sialostatin L, sialostatin L2 transcripts accumulate in the salivary glands during tick feeding (Kotsyfakis et al., 2007). Its target proteases are cathepsins L, V, S, and C with preferential affinity for cathepsins L and V (Kotsyfakis et al., 2007). Sialostatin L2 was shown to inhibit inflammasome formation during infection with $A$. phagocytophilum (Chen et al., 2014) via sialostatin L2-driven inhibition of caspase-1 maturation, leading to diminished IL- $1 \beta$ and IL-18 secretion by macrophages after stimulation with $A$. phagocytophilum (Chen et al., 2014). However, the mechanism was not due to direct caspase-1 or cathepsin L inhibition, but was 
instead dependent on reactive oxygen species (ROS) production by NADPH oxidase that was affected by the Loop2 domain of the cystatin (Chen et al., 2014). As mentioned above, sialostatin L2 interfered with JAK/STAT signaling in DC (Lieskovska et al., 2015b), attenuating STAT phosphorylation upon IFN- $\beta$ treatment and thus inhibiting the IFN- $\beta$ stimulated IP-10 and IRF7 chemokine genes (Lieskovska et al., 2015b). No interference with the IFN- $\beta$ receptor was observed, so the downstream components of the pathway were most likely affected. Moreover, this activity enhanced the replication of tick borne encephalitis virus in DC (Lieskovska et al., 2015b). Sialostatin L2 decreased the production of specific DC chemokines MIP- $1 \alpha$ and IP10 in response to Borrelia (Lieskovska et al., 2015a). Upon LTA/TLR2 stimulation of DC, sialostatin L2 attenuated Erk1/2 phosphorylation, inhibited the PI3K pathway by reducing Akt phosphorylation, and also reduced NF- $\mathrm{KB}$ phosphorylation. Impaired Erk1/2 phosphorylation was the only effect observed for sialostatin L2 after stimulation of DC with Borrelia spirochetes (Lieskovska et al., 2015a).

The role of sialostatin L2 in Borrelia transmission and tick feeding has also been addressed. RNAi of sialostatin L2 led to $40 \%$ mortality in tick feeding, reduced tick size, and reduced the number of eggs by about $70 \%$ (Kotsyfakis et al., 2007). Similar effects were seen when I. scapularis nymphs were exposed to guinea pigs immunized with sialostatin L2 (Kotsyfakis et al., 2008). The rejection rate of nymphs fed on immunized animals was three times higher compared to controls, and the time needed to finish a blood meal was prolonged by approximately 1 day (Kotsyfakis et al., 2008). Moreover, IgG isolated from immunized animals reduced sialostatin L2 inhibitory activity against cathepsin L (Kotsyfakis et al., 2008). Of note, sialostatin L2 has been referred to as a "silent antigen," meaning that corresponding antibodies cannot be found in naïve animals exposed to ticks despite an increased titer of specific antibodies in animals pre-immunized with recombinant protein. This can be explained by the amount of sialostatin L2 injected via the saliva into the host being too small to elicit a response (Kotsyfakis et al., 2008). Sialostatin L2 has also been shown to play an important role in Borrelia infection (Kotsyfakis et al., 2010). The skin of mice simultaneously injected with Borrelia and sialostatin L2 contained six-times more spirochetes than controls. Sialostatin L2 does not appear to bind spirochetes directly and had no effect on Borrelia growth in vitro, so the mechanism of Borrelia growth boost in skin remains unknown (Kotsyfakis et al., 2010).

\section{PROTEASE INHIBITORS AT THE TICK-HOST INTERFACE}

Tick cystatins and serpins can obviously affect many intracellular pathways and thus impair the functions of host immune cells. Moreover, they can also interfere with extracellular proteolysis, thereby inhibiting hemostasis (Figure 1). These activities take place at the site of attachment, where they cause local immunosuppression and inhibition of blood clotting. Of note, different inhibitors can cause similar phenotypes by targeting different pathways or even different components of the same pathway. Their actions are therefore redundant. Conversely, more than one effect is usually observed for a single inhibitor. Such concept of redundancy and pluripotency is probably a strategy developed by ticks during long-term co-evolution with their hosts (Chmelar et al., 2016). There is no doubt that salivary secretion at the tick-host interface is beneficial for the tick and deleterious for the host. From this perspective, tick inhibitors represent an important and interesting research field for the development of anti-tick vaccines and tick control strategies.

As shown on vaccination experiments, tick serpins and cystatins can contribute to the establishment of pathogens in the host (Imamura et al., 2008; Kotsyfakis et al., 2010). Such role of serpins is in accordance with observed positive effect of activated plasminogen activation system (PAS) with upregulated serpin PAI-2 on the establishment of Borrelia burgdorferi infection. The facilitation of infection resulted from direct enhancement of Borrelia dissemination and from the inhibition of inflammatory infiltration to the site of exposure (Haile et al., 2006). Borrelia recurrentis was shown to bind host serpin-C1 inhibitor-on its surface and thus inhibit complement activation (Grosskinsky et al., 2010). On contrary, mammalian cystatins were shown as regulators of cysteine proteases like cathepsin S and L, which contribute to the establishment of several viral infections (Kopitar-Jerala, 2012). Thus, the involvement of cystatins in the establishment of microbial and viral infection is not clear and cannot be easily addressed without experimental evidence.

\section{TICK PROTEASE INHIBITORS AS NOVEL DRUGS}

\section{Cystatins}

The inhibition of target proteases with tick-derived inhibitors can, however, be beneficial in different scenarios. Almost all the mammalian serine and cysteine proteases that are targets of tick inhibitors described in this review play important roles in various human diseases and pathologies. For a long time, the functions of lysosomal cysteine cathepsins (B, C, F, H, K, $\mathrm{L}, \mathrm{O}, \mathrm{S}, \mathrm{V}, \mathrm{X}$, and $\mathrm{W}$ ) were thought to be strictly limited to intracellular protein degradation and cellular metabolism. Recently, many cathepsins have been shown to be involved in multiple pathological processes. For example, increased serum levels of cathepsin L are associated with metastatic stage of different cancer types and poor patient prognosis (Tumminello et al., 1996; Chen et al., 2011). Tumor cells can produce high amounts of cathepsin L, leading to high serum level, which is considered as blood marker of cancer (Denhardt et al., 1987). High concentration of cathepsin L in tumor and its vicinity leads to extracellular matrix degradation, higher tumor invasiveness, and several cancer-related health complications (Sudhan and Siemann, 2015). Other cysteine cathepsins may also participate in tumor invasion and metastasis (Kuester et al., 2008; Tan et al., 2013), so cystatins are considered possible effectors that could block the deleterious activity of cysteine cathepsins in cancer (Cox, 2009; Hap et al., 2011). Cysteine cathepsins also contribute to neurodegenerative disorders such as Alzheimer's, Parkinson's, and Huntington's disease and amyotrophic lateral 


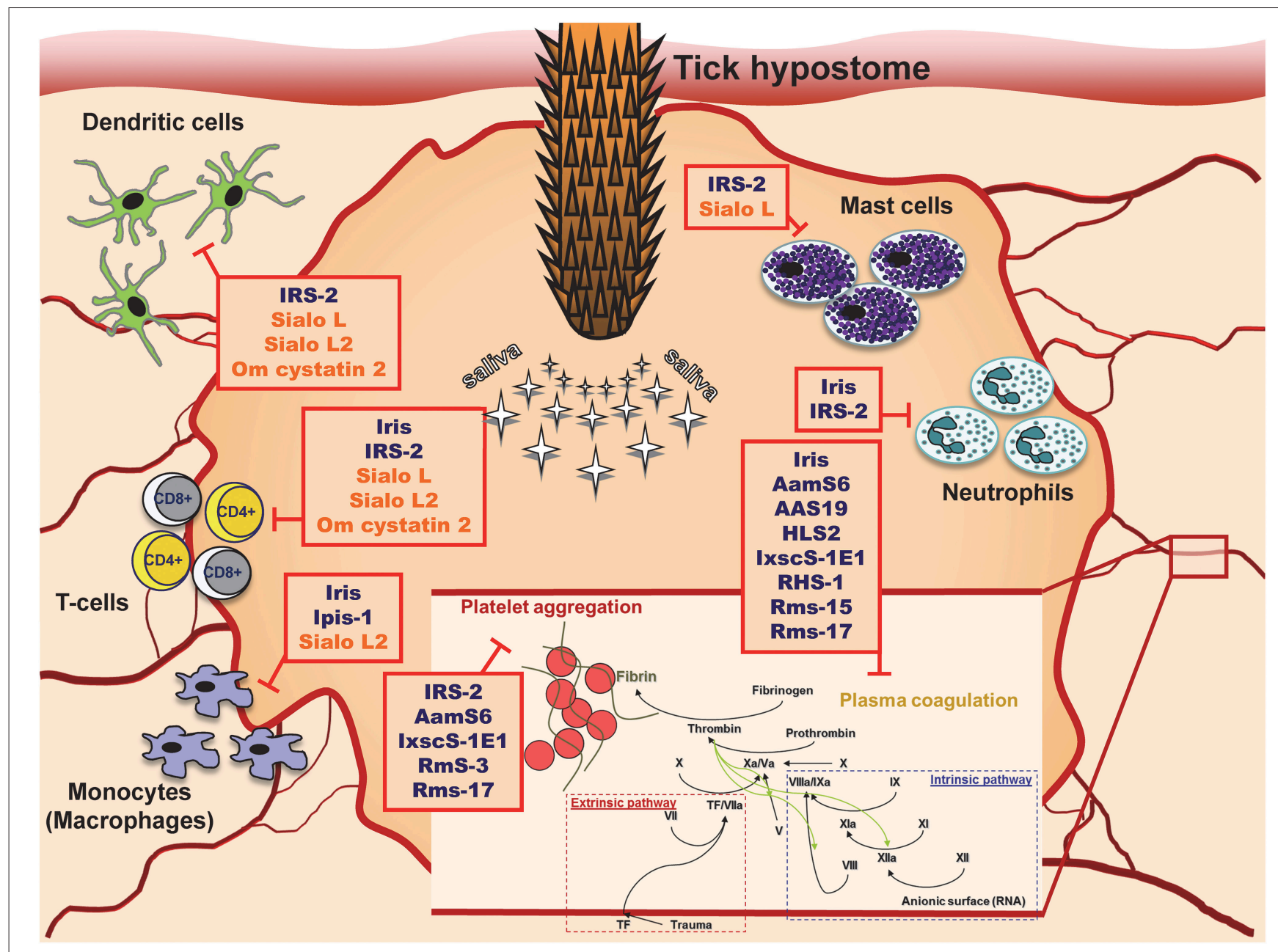

FIGURE 1 | Tick serpins and cystatins and their targets at the site of tick attachment.

sclerosis (Figure 2A; Pislar and Kos, 2014). The leakage of lysosomal cathepsins induces neuronal apoptosis and can also increase the inflammatory milieu in the central nervous system (Pislar and Kos, 2014). Cysteine cathepsins are also implicated in the pathogenesis of psoriasis (Kawada et al., 1997), muscular dystrophy (Takeda et al., 1992), abdominal aortic aneurysm and atherosclerosis (Liu et al., 2006), osteoporosis and rheumatoid arthritis (Yasuda et al., 2005), and acute pancreatitis (Halangk et al., 2000). Relatively recent data are accumulating to suggest that cysteine cathepsins are promising therapeutic targets (Kos et al., 2014; Sudhan and Siemann, 2015). The wide spectrum of tick cystatins with varying specificities provides an opportunity to take advantage of this rich source of natural cathepsin inhibitors.

\section{Serpins}

Serine proteases are best known as the building blocks of proteolytic cascades in the blood such as coagulation (Figure 1) or complement activation. The portfolio of their activities, however, is much wider. Neutrophils, mast cells, natural killer cells, and cytotoxic $\mathrm{T}$ cells all produce serine proteases responsible for extracellular matrix remodeling, microbe killing, cytokine activation, signaling via protease-activated receptors (PARs), or chemoattraction of leukocytes. As regulators of many processes, serine proteases often contribute to disease pathologies. Some diseases, in which serine proteases are implicated, are shown in Figure 2B. Signaling via PARs and the activation of coagulation in the tumor microenvironment link coagulation proteases with some of the complications seen in cancer (Shi et al., 2004; Han et al., 2011; Lima and Monteiro, 2013). Neutrophil proteases from azurophilic granules, namely cathepsin G, elastase, and protease 3 (PR3), play crucial roles in neutrophil anti-microbial activity and are indispensable for the clearance of some pathogens (Hahn et al., 2011; Steinwede et al., 2012). Many studies have also described neutrophil proteases as important regulators of inflammatory and immune processes (Pham, 2006, 2008), albeit with deleterious effects in some cases. For instance, due to the large amounts of elastin present in the lung connective tissue, lungs are very sensitive to dysregulation and/or increased levels of elastolytic proteases such as neutrophil elastase (Sandhaus and Turino, 

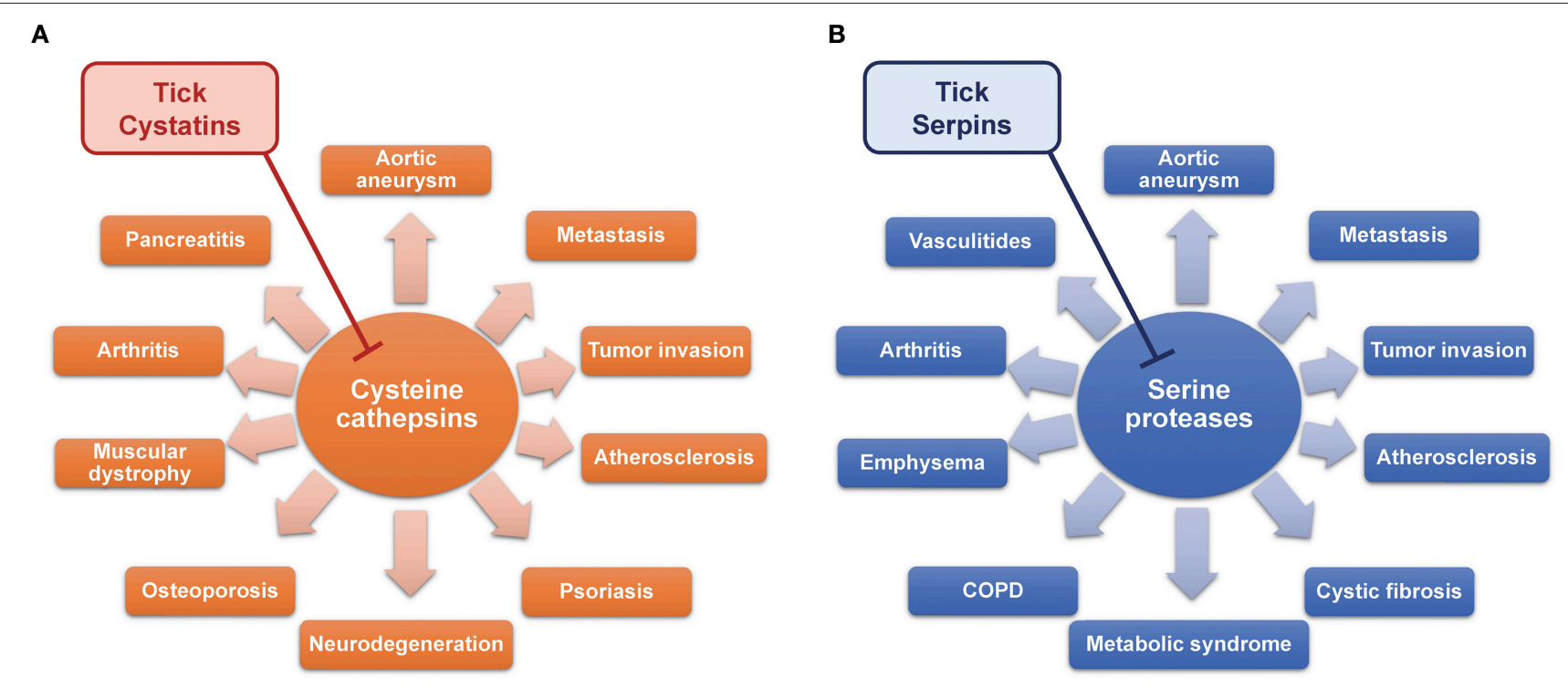

FIGURE 2 | The therapeutic potential of tick cystatins (A) and serpins (B) by inhibiting cysteine and serine proteases in various diseases.

2013), which results in several lung diseases. Elastase and cathepsin G facilitate the spreading of metastases to the lungs due to the degradation of antitumorigenic factor thrombospondin1 (El Rayes et al., 2015). Furthermore, neutrophil proteases have been implicated in the pathogenesis of cystic fibrosis (Twigg et al., 2015; Wagner et al., 2016), chronic obstructive pulmonary disease (COPD) (Shapiro, 2002; Owen, 2008), and emphysema (Ekeowa et al., 2009). In anti-neutrophil cytoplasmic autoantibody (ANCA)-associated vasculitides such as Wegener's granulomatosis, neutrophils are activated by auto-antibodies against PR3 (Niles et al., 1989), leading to the production of neutrophil extracellular traps (NETs) containing PR3 and to necrosis (Kessenbrock et al., 2009). Cathepsin G is chemotactic for monocytes in rheumatoid arthritis (Miyata et al., 2007), and the inhibition of neutrophil elastase improved some of the symptoms of this disease (Di Cesare Mannelli et al., 2016). Interestingly, obesity and metabolic syndrome also seem to be affected by neutrophil proteases (Talukdar et al., 2012; MansuyAubert et al., 2013). Mast cells are another significant source of several serine proteases, mainly chymases and tryptases, which are involved in extracellular matrix remodeling, chemoattraction of neutrophils, and protein processing and activation (Pejler et al., 2010). Mast cell chymase and tryptase have been shown to be involved in the pathogenesis of abdominal aortic aneurysm (Sun et al., 2009; Zhang et al., 2011) and atherosclerosis (Sun et al., 2007; Bot et al., 2015).

Due to these diverse and clinically relevant effects of serine proteases, their potential use as therapeutic targets is being thoroughly discussed by scientific community (Guay et al., 2006; Quinn et al., 2010; Caughey, 2016). Tick salivary glands express a large number of serine protease inhibitors with different specificities that could be used as novel drugs against malfunctioning proteases.

\section{CONCLUDING REMARKS}

Novel pharmacoactive compounds are being developed either by artificial synthesis or by isolating potential candidates from various organisms including parasites (Cherniack, 2011). For instance, hirudin (a thrombin inhibitor from leeches) and its congener bivalrudin have been useful in the treatment of blood coagulation disorders (Kennedy et al., 2012). Ticks are parasites that have evolved multiple ways to evade or manipulate host immune and hemostatic systems (Chmelar et al., 2012). Tick saliva contains hundreds of proteins not only with antihemostatic features (Maritz-Olivier et al., 2007) but also with anti-complement, anti-inflammatory, and immunomodulatory effects on the host (Kazimirova and Stibraniova, 2013).

As discussed in this review, salivary cystatins and serpins display such features and their functions have been studied thoroughly. Moreover, both superfamilies are represented in the vertebrate host and the functions of their members are often known. Therefore, we can predict at least to some degree, which processes or pathways will be targeted by tick proteins. An important advantage of cystatins and serpins is their functional specificity; for example, sialostatins $\mathrm{L}$ and L2 cause similar phenotypes (inhibition of IFN- $\beta$ signaling) either by inhibiting the IFN- $\beta$ production (sialostatin L) or by inhibiting STAT3 phosphorylation downstream from IFN- $\beta$ (sialostatin L2) (Lieskovska et al., 2015a,b). The possibility of targeting specific processes is crucial for the development of "patient-tailored" immunotherapeutic strategies (Scherer et al., 2010; Stephenson et al., 2016). Furthermore, tick cystatins and serpins are not the only families in ticks that deserve attention, since there are many tick-specific proteins secreted into the saliva of unknown function. Characterizing ticks using the transcriptomic approach has created a broad field and data 
repository, which we can search for novel drugs and potential therapeutics.

\section{AUTHOR CONTRIBUTIONS}

JC and JK wrote the manuscript, JK prepared the tables, JC prepared the figures, HL and $\mathrm{MK}$ edited and revised the manuscript.

\section{REFERENCES}

Amara, U., Rittirsch, D., Flierl, M., Bruckner, U., Klos, A., Gebhard, F., et al. (2008). Interaction between the coagulation and complement system. Adv. Exp. Med. Biol. 632, 71-79. doi: 10.1007/978-0-387-78952-1_6

Anguita, J., Ramamoorthi, N., Hovius, J. W., Das, S., Thomas, V., Persinski, R., et al. (2002). Salp15, an ixodes scapularis salivary protein, inhibits CD4(+) T cell activation. Immunity 16, 849-859. doi: 10.1016/S1074-7613(02)00325-4

Ayllon, N., Villar, M., Galindo, R. C., Kocan, K. M., Sima, R., Lopez, J. A., et al. (2015). Systems biology of tissue-specific response to Anaplasma phagocytophilum reveals differentiated apoptosis in the tick vector Ixodes scapularis. PLoS Genet. 11:e1005120. doi: 10.1371/journal.pgen.1005120

Bania, J., Stachowiak, D., and Polanowski, A. (1999). Primary structure and properties of the cathepsin G/chymotrypsin inhibitor from the larval hemolymph of Apis mellifera. Eur. J. Biochem. 262, 680-687. doi: 10.1046/j.1432-1327.1999.00406.x

Belorgey, D., Hagglof, P., Karlsson-Li, S., and Lomas, D. A. (2007). Protein misfolding and the serpinopathies. Prion 1, 15-20. doi: 10.4161/pri.1.1.3974

Bot, I., Shi, G. P., and Kovanen, P. T. (2015). Mast cells as effectors in atherosclerosis. Arterioscler. Thromb. Vasc. Biol. 35, 265-271. doi: 10.1161/ATVBAHA.114.303570

Brossard, M., and Wikel, S. K. (2004). Tick immunobiology. Parasitology 129 (Suppl.), S161-S176. doi: 10.1017/S0031182004004834

Calvo, E., Mizurini, D. M., Sa-Nunes, A., Ribeiro, J. M., Andersen, J. F., Mans, B. J., et al. (2011). Alboserpin, a factor Xa inhibitor from the mosquito vector of yellow fever, binds heparin and membrane phospholipids and exhibits antithrombotic activity. J. Biol. Chem. 286, 27998-28010. doi: $10.1074 /$ jbc.M111.247924

Carrell, R. W., and Read, R. J. (2016). How serpins transport hormones and regulate their release. Semin. Cell Dev. Biol. 62, 133-141. doi: 10.1016/j.semcdb. 2016.12.007

Caughey, G. H. (2016). Mast cell proteases as pharmacological targets. Eur. J. Pharmacol. 778, 44-55. doi: 10.1016/j.ejphar.2015.04.045

Chalaire, K. C., Kim, T. K., Garcia-Rodriguez, H., and Mulenga, A. (2011). Amblyomma americanum (L.) (Acari: Ixodidae) tick salivary gland serine protease inhibitor (serpin) 6 is secreted into tick saliva during tick feeding. J. Exp. Biol. 214, 665-673. doi: 10.1242/jeb.052076

Chen, G., Wang, X., Severo, M. S., Sakhon, O. S., Sohail, M., Brown, L. J., et al. (2014). The tick salivary protein sialostatin L2 inhibits caspase-1-mediated inflammation during Anaplasma phagocytophilum infection. Infect. Immun. 82, 2553-2564. doi: 10.1128/IAI.01679-14

Chen, Q., Fei, J., Wu, L., Jiang, Z., Wu, Y., Zheng, Y., et al. (2011). Detection of cathepsin B, cathepsin L, cystatin C, urokinase plasminogen activator and urokinase plasminogen activator receptor in the sera of lung cancer patients. Oncol. Lett. 2, 693-699. doi: 10.3892/ol.2011.302

Cherniack, E. P. (2011). Bugs as drugs, part two: worms, leeches, scorpions, snails, ticks, centipedes, and spiders. Altern. Med. Rev. 16, 50-58.

Chmelar, J., Calvo, E., Pedra, J. H., Francischetti, I. M., and Kotsyfakis, M. (2012). Tick salivary secretion as a source of antihemostatics. J. Proteomics 75 , 3842-3854. doi: 10.1016/j.jprot.2012.04.026

Chmelar, J., Kotal, J., Kopecky, J., Pedra, J. H., and Kotsyfakis, M. (2016). All for one and one for all on the tick-host battlefield. Trends Parasitol. 32, 368-377. doi: $10.1016 /$ j.pt.2016.01.004

Chmelar, J., Oliveira, C. J., Rezacova, P., Francischetti, I. M., Kovarova, Z., Pejler, G., et al. (2011). A tick salivary protein targets cathepsin G and chymase

\section{FUNDING}

This work was supported by Grant agency of the Czech Republic (grant 16-07117Y to JC), by Grant agency of the University of South Bohemia to JK (grant 038/2016/P) and by intramural institutional support from the Institute of Parasitology, Biology Center of the Czech Academy of Sciences [RVO 60077344] to MK and $\mathrm{JK}$.

and inhibits host inflammation and platelet aggregation. Blood 117, 736-744. doi: 10.1182/blood-2010-06-293241

Colinet, D., Dubuffet, A., Cazes, D., Moreau, S., Drezen, J. M., and Poirie, M. (2009). A serpin from the parasitoid wasp Leptopilina boulardi targets the Drosophila phenoloxidase cascade. Dev. Comp. Immunol. 33, 681-689. doi: 10.1016/j.dci.2008.11.013

Corral-Rodriguez, M. A., Macedo-Ribeiro, S., Barbosa Pereira, P. J., and Fuentes-Prior, P. (2009). Tick-derived Kunitz-type inhibitors as antihemostatic factors. Insect Biochem. Mol. Biol. 39, 579-595. doi: 10.1016/j.ibmb.2009. 07.003

Cox, J. L. (2009). Cystatins and cancer. Front. Biosci. (Landmark Ed) 14, 463-474. doi: $10.2741 / 3255$

Dai, S. X., Zhang, A. D., and Huang, J. F. (2012). Evolution, expansion and expression of the Kunitz/BPTI gene family associated with long-term blood feeding in Ixodes Scapularis. BMC Evol. Biol. 12:4. doi: 10.1186/1471-2148-12-4

Davis, R. L., Shrimpton, A. E., Holohan, P. D., Bradshaw, C., Feiglin, D., Collins, G. H., et al. (1999). Familial dementia caused by polymerization of mutant neuroserpin. Nature 401, 376-379. doi: 10.1038/43894

Denhardt, D. T., Greenberg, A. H., Egan, S. E., Hamilton, R. T., and Wright, J. A. (1987). Cysteine proteinase cathepsin L expression correlates closely with the metastatic potential of H-ras-transformed murine fibroblasts. Oncogene 2, 55-59.

Di Cesare Mannelli, L., Micheli, L., Cinci, L., Maresca, M., Vergelli, C., Pacini, A., et al. (2016). Effects of the neutrophil elastase inhibitor EL17 in rat adjuvant-induced arthritis. Rheumatology (Oxford) 55, 1285-1294. doi: 10.1093/rheumatology/kew055

Ekeowa, U. I., Gooptu, B., Belorgey, D., Hagglof, P., Karlsson-Li, S., Miranda, E., et al. (2009). alphal-Antitrypsin deficiency, chronic obstructive pulmonary disease and the serpinopathies. Clin. Sci. 116, 837-850. doi: 10.1042/CS20080484

El Rayes, T., Catena, R., Lee, S., Stawowczyk, M., Joshi, N., Fischbach, C., et al. (2015). Lung inflammation promotes metastasis through neutrophil proteasemediated degradation of Tsp-1. Proc. Natl. Acad. Sci. U.S.A. 112, 16000-16005. doi: 10.1073/pnas.1507294112

Fogaca, A. C., Almeida, I. C., Eberlin, M. N., Tanaka, A. S., Bulet, P., and Daffre, S. (2006). Ixodidin, a novel antimicrobial peptide from the hemocytes of the cattle tick Boophilus microplus with inhibitory activity against serine proteinases. Peptides 27, 667-674. doi: 10.1016/j.peptides.2005.07.013

Frazao, B., Vasconcelos, V., and Antunes, A. (2012). Sea anemone (Cnidaria, Anthozoa, Actiniaria) toxins: an overview. Mar. Drugs 10, 1812-1851. doi: $10.3390 / \mathrm{md} 10081812$

Grosskinsky, S., Schott, M., Brenner, C., Cutler, S. J., Simon, M. M., and Wallich, R. (2010). Human complement regulators C4b-binding protein and C1 esterase inhibitor interact with a novel outer surface protein of Borrelia recurrentis. PLoS Negl. Trop. Dis. 4:e698. doi: 10.1371/journal.pntd.0000698

Grunclova, L., Horn, M., Vancova, M., Sojka, D., Franta, Z., Mares, M., et al. (2006). Two secreted cystatins of the soft tick Ornithodoros moubata: differential expression pattern and inhibitory specificity. Biol. Chem. 387, 1635-1644. doi: 10.1515/BC.2006.204

Guay, C., Laviolette, M., and Tremblay, G. M. (2006). Targeting serine proteases in asthma. Curr. Top. Med. Chem. 6, 393-402. doi: 10.2174/156802606776287054

Gulia-Nuss, M., Nuss, A. B., Meyer, J. M., Sonenshine, D. E., Roe, R. M., Waterhouse, R. M., et al. (2016). Genomic insights into the Ixodes scapularis tick vector of Lyme disease. Nat. Commun. 7:10507. doi: 10.1038/ncomms10507 
Gulley, M. M., Zhang, X., and Michel, K. (2013). The roles of serpins in mosquito immunology and physiology. J. Insect Physiol. 59, 138-147. doi: 10.1016/j.jinsphys.2012.08.015

Hahn, I., Klaus, A., Janze, A. K., Steinwede, K., Ding, N., Bohling, J., et al. (2011). Cathepsin $G$ and neutrophil elastase play critical and nonredundant roles in lung-protective immunity against Streptococcus pneumoniae in mice. Infect. Immun. 79, 4893-4901. doi: 10.1128/IAI.05593-11

Haile, W. B., Coleman, J. L., and Benach, J. L. (2006). Reciprocal upregulation of urokinase plasminogen activator and its inhibitor, PAI-2, by Borrelia burgdorferi affects bacterial penetration and host-inflammatory response. Cell. Microbiol. 8, 1349-1360. doi: 10.1111/j.1462-5822.2006.00717.x

Halangk, W., Lerch, M. M., Brandt-Nedelev, B., Roth, W., Ruthenbuerger, M., Reinheckel, T., et al. (2000). Role of cathepsin B in intracellular trypsinogen activation and the onset of acute pancreatitis. J. Clin. Invest. 106, 773-781. doi: 10.1172/JCI9411

Han, N., Jin, K., He, K., Cao, J., and Teng, L. (2011). Protease-activated receptors in cancer: a systematic review. Oncol. Lett. 2, 599-608. doi: 10.3892/ol.2011.291

Hap, A., Kielan, W., Grzebieniak, Z., Siewinski, M., Rudnicki, J., Tarnawa, R., et al. (2011). Control of active B and L cathepsins in tissues of colorectal cancer using cystatins isolated from chicken egg proteins: in vitro studies. Folia Histochem. Cytobiol. 49, 670-676. doi: 10.5603/FHC.2011.0075

Heit, C., Jackson, B. C., Mcandrews, M., Wright, M. W., Thompson, D. C., Silverman, G. A., et al. (2013). Update of the human and mouse SERPIN gene superfamily. Hum. Genomics 7:22. doi: 10.1186/1479-7364-7-22

Horka, H., Staudt, V., Klein, M., Taube, C., Reuter, S., Dehzad, N., et al. (2012). The tick salivary protein sialostatin L inhibits the Th9-derived production of the asthma-promoting cytokine IL-9 and is effective in the prevention of experimental asthma. J. Immunol. 188, 2669-2676. doi: 10.4049/jimmunol.1100529

Horn, M., Nussbaumerova, M., Sanda, M., Kovarova, Z., Srba, J., Franta, Z., et al. (2009). Hemoglobin digestion in blood-feeding ticks: mapping a multipeptidase pathway by functional proteomics. Chem. Biol. 16, 1053-1063. doi: 10.1016/j.chembiol.2009.09.009

Huntington, J. A., and Li, W. (2009). Structural insights into the multiple functions of protein C inhibitor. Cell. Mol. Life Sci. 66, 113-121. doi: 10.1007/s00018-008-8371-0

Ibelli, A. M., Hermance, M. M., Kim, T. K., Gonzalez, C. L., and Mulenga, A. (2013). Bioinformatics and expression analyses of the Ixodes scapularis tick cystatin family. Exp. Appl. Acarol. 60, 41-53. doi: 10.1007/s10493-012-9613-2

Ibelli, A. M., Kim, T. K., Hill, C. C., Lewis, L. A., Bakshi, M., Miller, S., et al. (2014). A blood meal-induced Ixodes scapularis tick saliva serpin inhibits trypsin and thrombin, and interferes with platelet aggregation and blood clotting. Int. J. Parasitol. 44, 369-379. doi: 10.1016/j.ijpara.2014.01.010

Imamura, S., Da Silva Vaz Junior, I., Sugino, M., Ohashi, K., and Onuma, M. (2005). A serine protease inhibitor (serpin) from Haemaphysalis longicornis as an anti-tick vaccine. Vaccine 23, 1301-1311. doi: 10.1016/j.vaccine.2004.08.041

Imamura, S., Konnai, S., Vaz Ida, S., Yamada, S., Nakajima, C., Ito, Y., et al. (2008). Effects of anti-tick cocktail vaccine against Rhipicephalus appendiculatus. Jpn. J. Vet. Res. 56, 85-98. doi: 10.14943/jjvr.56.2.85

Imamura, S., Konnai, S., Yamada, S., Parizi, L. F., Githaka, N., Vaz Ida, S. Jr., et al. (2013). Identification and partial characterization of a gut Rhipicephalus appendiculatus cystatin. Ticks Tick Borne Dis. 4, 138-144. doi: 10.1016/j.ttbdis.2012.11.001

Imamura, S., Namangala, B., Tajima, T., Tembo, M. E., Yasuda, J., Ohashi, K., et al. (2006). Two serine protease inhibitors (serpins) that induce a bovine protective immune response against Rhipicephalus appendiculatus ticks. Vaccine 24, 2230-2237. doi: 10.1016/j.vaccine.2005.10.055

Jin, C., and Flavell, R. A. (2010). Molecular mechanism of NLRP3 inflammasome activation. J. Clin. Immunol. 30, 628-631. doi: 10.1007/s10875-010-9440-3

Jittapalapong, S., Kaewhom, P., Pumhom, P., Canales, M., De La Fuente, J., and Stich, R. W. (2010). Immunization of rabbits with recombinant serine protease inhibitor reduces the performance of adult female Rhipicephalus microplus. Transbound. Emerg. Dis. 57, 103-106. doi: 10.1111/j.1865-1682.2010.01108.x

Kaewhom, P., Sirinarumitr, T., Chantakru, S., and Jittapalapong, S. (2007). Cloning and characterization of cDNA encoding a serine protease inhibitor from salivary glands of thai cattle tick (Boophilus microplus). Kasetsart J. (Nat. Sci.) $41,74-80$.
Kanost, M. R. (1999). Serine proteinase inhibitors in arthropod immunity. Dev. Comp. Immunol. 23, 291-301. doi: 10.1016/S0145-305X(99)00012-9

Karim, S., Miller, N. J., Valenzuela, J., Sauer, J. R., and Mather, T. N. (2005). RNAi-mediated gene silencing to assess the role of synaptobrevin and cystatin in tick blood feeding. Biochem. Biophys. Res. Commun. 334, 1336-1342. doi: 10.1016/j.bbrc.2005.07.036

Karim, S., and Ribeiro, J. M. (2015). An Insight into the Sialome of the Lone Star Tick, Amblyomma americanum, with a Glimpse on its time dependent gene expression. PLoS ONE 10:e0131292. doi: 10.1371/journal.pone.0131292

Karim, S., Singh, P., and Ribeiro, J. M. (2011). A deep insight into the sialotranscriptome of the gulf coast tick, Amblyomma maculatum. PLoS ONE 6:e28525. doi: 10.1371/journal.pone.0028525

Kawada, A., Hara, K., Kominami, E., Hiruma, M., Noguchi, H., and Ishibashi, A. (1997). Processing of cathepsins L, B and D in psoriatic epidermis. Arch. Dermatol. Res. 289, 87-93. doi: 10.1007/s004030050160

Kazimirova, M., and Stibraniova, I. (2013). Tick salivary compounds: their role in modulation of host defences and pathogen transmission. Front. Cell. Infect. Microbiol. 3:43. doi: 10.3389/fcimb.2013.00043

Kennedy, B., Gargoum, F. S., Kennedy, L., Khan, F., Curran, D. R., and O'connor, T. M. (2012). Emerging anticoagulants. Curr. Med. Chem. 19, 3388-3416. doi: 10.2174/092986712801215847

Kessenbrock, K., Krumbholz, M., Schonermarck, U., Back, W., Gross, W. L., Werb, Z., et al. (2009). Netting neutrophils in autoimmune small-vessel vasculitis. Nat. Med. 15, 623-625. doi: 10.1038/nm.1959

Kim, H. S., Krege, J. H., Kluckman, K. D., Hagaman, J. R., Hodgin, J. B., Best, C. F., et al. (1995). Genetic control of blood pressure and the angiotensinogen locus. Proc. Natl. Acad. Sci. U.S.A. 92, 2735-2739. doi: 10.1073/pnas.92.7.2735

Kim, T. K., Radulovic, Z., and Mulenga, A. (2016). Target validation of highly conserved Amblyomma americanum tick saliva serine protease inhibitor 19 Ticks Tick Borne Dis. 7, 405-414. doi: 10.1016/j.ttbdis.2015.12.017

Kim, T. K., Tirloni, L., Radulovic, Z., Lewis, L., Bakshi, M., Hill, C., et al. (2015). Conserved Amblyomma americanum tick Serpin19, an inhibitor of blood clotting factors Xa and XIa, trypsin and plasmin, has anti-haemostatic functions. Int. J. Parasitol. 45, 613-627. doi: 10.1016/j.ijpara.2015.03.009

Klein, M., Bruhl, T. J., Staudt, V., Reuter, S., Grebe, N., Gerlitzki, B., et al. (2015). Tick salivary sialostatin $\mathrm{L}$ represses the initiation of immune responses by targeting IRF4-dependent transcription in murine mast cells. J. Immunol. 195, 621-631. doi: 10.4049/jimmunol.1401823

Kopitar-Jerala, N. (2012). The role of cysteine proteinases and their inhibitors in the host-pathogen cross talk. Curr. Protein Pept. Sci. 13, 767-775. doi: $10.2174 / 138920312804871102$

Kordis, D., and Turk, V. (2009). Phylogenomic analysis of the cystatin superfamily in eukaryotes and prokaryotes. BMC Evol. Biol. 9:266. doi: 10.1186/1471-2148-9-266

Kos, J., Mitrovic, A., and Mirkovic, B. (2014). The current stage of cathepsin B inhibitors as potential anticancer agents. Future Med. Chem. 6, 1355-1371. doi: 10.4155/fmc. 14.73

Kotsyfakis, M., Anderson, J. M., Andersen, J. F., Calvo, E., Francischetti, I. M., Mather, T. N., et al. (2008). Cutting edge: immunity against a "silent" salivary antigen of the Lyme vector Ixodes scapularis impairs its ability to feed. J. Immunol. 181, 5209-5212. doi: 10.4049/jimmunol.181.8.5209

Kotsyfakis, M., Horka, H., Salat, J., and Andersen, J. F. (2010). The crystal structures of two salivary cystatins from the tick Ixodes scapularis and the effect of these inhibitors on the establishment of Borrelia burgdorferi infection in a murine model. Mol. Microbiol. 77, 456-470. doi: 10.1111/j.1365-2958.2010.07220.x

Kotsyfakis, M., Karim, S., Andersen, J. F., Mather, T. N., and Ribeiro, J. M. (2007). Selective cysteine protease inhibition contributes to blood-feeding success of the tick Ixodes scapularis. J. Biol. Chem. 282, 29256-29263. doi: 10.1074/jbc.M703143200

Kotsyfakis, M., Kopacek, P., Franta, Z., Pedra, J. H., and Ribeiro, J. M. (2015a). Deep Sequencing Analysis of the Ixodes ricinus Haemocytome. PLoS Negl. Trop. Dis. 9:e0003754. doi: 10.1371/journal.pntd.0003754

Kotsyfakis, M., Sa-Nunes, A., Francischetti, I. M., Mather, T. N., Andersen, J. F., and Ribeiro, J. M. (2006). Antiinflammatory and immunosuppressive activity of sialostatin L, a salivary cystatin from the tick Ixodes scapularis. J. Biol. Chem. 281, 26298-26307. doi: 10.1074/jbc.M513010200 
Kotsyfakis, M., Schwarz, A., Erhart, J., and Ribeiro, J. M. (2015b). Tissue- and timedependent transcription in Ixodes ricinus salivary glands and midguts when blood feeding on the vertebrate host. Sci. Rep. 5:9103. doi: 10.1038/srep09103

Kovarova, Z., Chmelar, J., Sanda, M., Brynda, J., Mares, M., and Rezacova, P. (2010). Crystallization and diffraction analysis of the serpin IRS-2 from the hard tick Ixodes ricinus. Acta Crystallogr. Sect. F Struct. Biol. Cryst. Commun. 66, 1453-1457. doi: 10.1107/S1744309110032343

Kuester, D., Lippert, H., Roessner, A., and Krueger, S. (2008). The cathepsin family and their role in colorectal cancer. Pathol. Res. Pract. 204, 491-500. doi: 10.1016/j.prp.2008.04.010

Law, R. H., Zhang, Q., Mcgowan, S., Buckle, A. M., Silverman, G. A., Wong, W., et al. (2006). An overview of the serpin superfamily. Genome Biol. 7:216. doi: 10.1186/gb-2006-7-5-216

Leboulle, G., Crippa, M., Decrem, Y., Mejri, N., Brossard, M., Bollen, A., et al. (2002a). Characterization of a novel salivary immunosuppressive protein from Ixodes ricinus ticks. J. Biol. Chem. 277, 10083-10089. doi: 10.1074/jbc.M111391200

Leboulle, G., Rochez, C., Louahed, J., Ruti, B., Brossard, M., Bollen, A., et al. (2002b). Isolation of Ixodes ricinus salivary gland mRNA encoding factors induced during blood feeding. Am. J. Trop. Med. Hyg. 66, 225-233. doi: $10.4269 /$ ajtmh.2002.66.225

Li, J., Zhang, C., Xu, X., Wang, J., Yu, H., Lai, R., et al. (2007). Trypsin inhibitory loop is an excellent lead structure to design serine protease inhibitors and antimicrobial peptides. FASEB J. 21, 2466-2473. doi: 10.1096/fj.06-7966com

Lieskovska, J., Palenikova, J., Langhansova, H., Campos Chagas, A., Calvo, E., Kotsyfakis, M., et al. (2015a). Tick sialostatins L and L2 differentially influence dendritic cell responses to Borrelia spirochetes. Parasit. Vectors 8:275. doi: 10.1186/s13071-015-0887-1

Lieskovska, J., Palenikova, J., Sirmarova, J., Elsterova, J., Kotsyfakis, M., Campos Chagas, A., et al. (2015b). Tick salivary cystatin sialostatin L2 suppresses IFN responses in mouse dendritic cells. Parasite Immunol. 37, 70-78. doi: $10.1111 /$ pim. 12162

Lima, C. A., Sasaki, S. D., and Tanaka, A. S. (2006). Bmcystatin, a cysteine proteinase inhibitor characterized from the tick Boophilus microplus. Biochem. Biophys. Res. Commun. 347, 44-50. doi: 10.1016/j.bbrc.2006.06.018

Lima, L. G., and Monteiro, R. Q. (2013). Activation of blood coagulation in cancer: implications for tumour progression. Biosci. Rep. 33:pii: e00064. doi: 10.1042/BSR20130057

Liu, J., Sukhova, G. K., Yang, J. T., Sun, J., Ma, L., Ren, A., et al. (2006). Cathepsin L expression and regulation in human abdominal aortic aneurysm, atherosclerosis, and vascular cells. Atherosclerosis 184, 302-311. doi: 10.1016/j.atherosclerosis.2005.05.012

Liu, X. Y., De La Fuente, J., Cote, M., Galindo, R. C., Moutailler, S., VayssierTaussat, M., et al. (2014). IrSPI, a tick serine protease inhibitor involved in tick feeding and Bartonella henselae infection. PLoS Negl. Trop. Dis. 8:e2993. doi: 10.1371/journal.pntd.0002993

Lomas, D. A., Hurst, J. R., and Gooptu, B. (2016). Update on alpha1 antitrypsin deficiency: new therapies. J. Hepatol. 65, 413-424. doi: 10.1016/j.jhep.2016.03.010

Lu, H., Cassis, L. A., Kooi, C. W., and Daugherty, A. (2016). Structure and functions of angiotensinogen. Hypertens. Res. 39, 492-500. doi: $10.1038 / \mathrm{hr} .2016 .17$

Lu, S., Soares, T. S., Vaz Junior, I. S., Lovato, D. V., and Tanaka, A. S. (2014). Rmcystatin3, a cysteine protease inhibitor from Rhipicephalus microplus hemocytes involved in immune response. Biochimie 106, 17-23. doi: 10.1016/j.biochi.2014.07.012

Mansuy-Aubert, V., Zhou, Q. L., Xie, X., Gong, Z., Huang, J. Y., Khan, A. R., et al. (2013). Imbalance between neutrophil elastase and its inhibitor alpha1antitrypsin in obesity alters insulin sensitivity, inflammation, and energy expenditure. Cell Metab. 17, 534-548. doi: 10.1016/j.cmet.2013.03.005

Maritz-Olivier, C., Stutzer, C., Jongejan, F., Neitz, A. W., and Gaspar, A. R. (2007). Tick anti-hemostatics: targets for future vaccines and therapeutics. Trends Parasitol. 23, 397-407. doi: 10.1016/j.pt.2007.07.005

Meekins, D. A., Kanost, M. R., and Michel, K. (2017). Serpins in arthropod biology. Semin. Cell Dev. Biol. 62, 105-119. doi: 10.1016/j.semcdb.2016. 09.001

Miyata, J., Tani, K., Sato, K., Otsuka, S., Urata, T., Lkhagvaa, B., et al. (2007). Cathepsin G: the significance in rheumatoid arthritis as a monocyte chemoattractant. Rheumatol Int. 27, 375-382. doi: 10.1007/s00296-0060210-8

Moreira, H. N., Barcelos, R. M., Vidigal, P. M., Klein, R. C., Montandon, C. E., Maciel, T. E., et al. (2017). A deep insight into the whole transcriptome of midguts, ovaries and salivary glands of the Amblyomma sculptum tick. Parasitol. Int. 66, 64-73. doi: 10.1016/j.parint.2016.10.011

Mulenga, A., Blandon, M., and Khumthong, R. (2007a). The molecular basis of the Amblyomma americanum tick attachment phase. Exp. Appl. Acarol. 41, 267-287. doi: 10.1007/s10493-007-9064-3

Mulenga, A., Khumthong, R., and Blandon, M. A. (2007b). Molecular and expression analysis of a family of the Amblyomma americanum tick Lospins. J. Exp. Biol. 210, 3188-3198. doi: 10.1242/jeb.006494

Mulenga, A., Khumthong, R., and Chalaire, K. C. (2009). Ixodes scapularis tick serine proteinase inhibitor (serpin) gene family; annotation and transcriptional analysis. BMC Genomics 10:217. doi: 10.1186/1471-2164$10-217$

Mulenga, A., Khumthong, R., Chalaire, K. C., Strey, O., and Teel, P. (2008). Molecular and biological characterization of the Amblyomma americanum organic anion transporter polypeptide. J. Exp. Biol. 211, 3401-3408. doi: $10.1242 /$ jeb.022376

Mulenga, A., Kim, T., and Ibelli, A. M. (2013). Amblyomma americanum tick saliva serine protease inhibitor 6 is a cross-class inhibitor of serine proteases and papain-like cysteine proteases that delays plasma clotting and inhibits platelet aggregation. Insect. Mol. Biol. 22, 306-319. doi: 10.1111/imb. 12024

Mulenga, A., Tsuda, A., Onuma, M., and Sugimoto, C. (2003). Four serine proteinase inhibitors (serpin) from the brown ear tick, Rhiphicephalus appendiculatus; cDNA cloning and preliminary characterization. Insect Biochem. Mol. Biol. 33, 267-276. doi: 10.1016/S0965-1748(02) 00240-0

Muller, S., Dennemarker, J., and Reinheckel, T. (2012). Specific functions of lysosomal proteases in endocytic and autophagic pathways. Biochim. Biophys. Acta 1824, 34-43. doi: 10.1016/j.bbapap.2011.07.003

Nickel, W. (2003). The mystery of nonclassical protein secretion. A current view on cargo proteins and potential export routes. Eur. J. Biochem. 270, 2109-2119. doi: 10.1046/j.1432-1033.2003.03577.x

Niles, J. L., Mccluskey, R. T., Ahmad, M. F., and Arnaout, M. A. (1989). Wegener's granulomatosis autoantigen is a novel neutrophil serine proteinase. Blood 74, 1888-1893.

Ooi, C. P., Haines, L. R., Southern, D. M., Lehane, M. J., and Acosta-Serrano, A. (2015). Tsetse GmmSRPN10 has anti-complement activity and is important for successful establishment of trypanosome infections in the fly midgut. PLoS Negl. Trop. Dis. 9:e3448. doi: 10.1371/journal.pntd.0003448

Owen, C. A. (2008). Roles for proteinases in the pathogenesis of chronic obstructive pulmonary disease. Int. J. Chron. Obstruct. Pulmon. Dis. 3, 253-268. doi: 10.2147/COPD.S2089

Pal, U., Li, X., Wang, T., Montgomery, R. R., Ramamoorthi, N., Desilva, A. M., et al. (2004). TROSPA, an Ixodes scapularis receptor for Borrelia burgdorferi. Cell 119, 457-468. doi: 10.1016/j.cell.2004.10.027

Palenikova, J., Lieskovska, J., Langhansova, H., Kotsyfakis, M., Chmelar, J., and Kopecky, J. (2015). Ixodes ricinus salivary serpin IRS-2 affects Th17 differentiation via inhibition of the interleukin-6/STAT-3 signaling pathway. Infect. Immun. 83, 1949-1956. doi: 10.1128/IAI.03065-14

Parizi, L. F., Sabadin, G. A., Alzugaray, M. F., Seixas, A., Logullo, C., Konnai, S., et al. (2015). Rhipicephalus microplus and Ixodes ovatus cystatins in tick blood digestion and evasion of host immune response. Parasit. Vectors 8:122. doi: 10.1186/s13071-015-0743-3

Pejler, G., Ronnberg, E., Waern, I., and Wernersson, S. (2010). Mast cell proteases: multifaceted regulators of inflammatory disease. Blood 115, 4981-4990. doi: 10.1182/blood-2010-01-257287

Perner, J., Provaznik, J., Schrenkova, J., Urbanova, V., Ribeiro, J. M., and Kopacek, P. (2016). RNA-seq analyses of the midgut from blood- and serum-fed Ixodes ricinus ticks. Sci. Rep. 6:36695. doi: 10.1038/srep36695

Pham, C. T. (2006). Neutrophil serine proteases: specific regulators of inflammation. Nat. Rev. Immunol. 6, 541-550. doi: 10.1038/nri1841

Pham, C. T. (2008). Neutrophil serine proteases fine-tune the inflammatory response. Int. J. Biochem. Cell Biol. 40, 1317-1333. doi: 10.1016/j.biocel.2007.11.008 
Pierre, P., and Mellman, I. (1998). Developmental regulation of invariant chain proteolysis controls MHC class II trafficking in mouse dendritic cells. Cell 93, 1135-1145. doi: 10.1016/S0092-8674(00)81458-0

Pislar, A., and Kos, J. (2014). Cysteine cathepsins in neurological disorders. Mol. Neurobiol. 49, 1017-1030. doi: 10.1007/s12035-013-8576-6

Porter, L. M., Radulovic, Z. M., and Mulenga, A. (2017). A repertoire of protease inhibitor families in Amblyomma americanum and other tick species: inter-species comparative analyses. Parasit. Vectors 10:152. doi: 10.1186/s13071-017-2080-1

Porter, L., Radulovic, Z., Kim, T., Braz, G. R., Da Silva Vaz, I. Jr., and Mulenga, A. (2015). Bioinformatic analyses of male and female Amblyomma americanum tick expressed serine protease inhibitors (serpins). Ticks Tick Borne Dis. 6, 16-30. doi: 10.1016/j.ttbdis.2014.08.002

Prevot, P. P., Adam, B., Boudjeltia, K. Z., Brossard, M., Lins, L., Cauchie, P., et al. (2006). Anti-hemostatic effects of a serpin from the saliva of the tick Ixodes ricinus. J. Biol. Chem. 281, 26361-26369. doi: 10.1074/jbc.M604197200

Prevot, P. P., Beschin, A., Lins, L., Beaufays, J., Grosjean, A., Bruys, L., et al. (2009). Exosites mediate the anti-inflammatory effects of a multifunctional serpin from the saliva of the tick Ixodes ricinus. FEBS J. 276, 3235-3246. doi: 10.1111/j.1742-4658.2009.07038.x

Prevot, P. P., Couvreur, B., Denis, V., Brossard, M., Vanhamme, L., and Godfroid, E. (2007). Protective immunity against Ixodes ricinus induced by a salivary serpin. Vaccine 25, 3284-3292. doi: 10.1016/j.vaccine.2007.01.008

Quinn, D. J., Weldon, S., and Taggart, C. C. (2010). Antiproteases as therapeutics to target inflammation in cystic fibrosis. Open Respir. Med. J. 4, 20-31. doi: 10.2174/1874306401004010020

Rangel, C. K., Parizi, L. F., Sabadin, G. A., Costa, E. P., Romeiro, N. C., Isezaki, M., et al. (2017). Molecular and structural characterization of novel cystatins from the taiga tick Ixodes persulcatus. Ticks Tick Borne Dis. 8, 432-441. doi: 10.1016/j.ttbdis.2017.01.007

Rawlings, N. D., and Barrett, A. J. (1990). Evolution of proteins of the cystatin superfamily. J. Mol. Evol. 30, 60-71. doi: 10.1007/BF02102453

Rawlings, N. D., and Salvesen, G. (2013). Handbook of Proteolytic Enzymes, 3rd $E d n$. London; Boston: Academic Press.

Ribeiro, J. M., Slovak, M., and Francischetti, I. M. (2017). An insight into the sialome of Hyalomma excavatum. Ticks Tick Borne Dis. 8, 201-207. doi: 10.1016/j.ttbdis.2016.08.011

Rimphanitchayakit, V., and Tassanakajon, A. (2010). Structure and function of invertebrate Kazal-type serine proteinase inhibitors. Dev. Comp. Immunol. 34, 377-386. doi: 10.1016/j.dci.2009.12.004

Rodriguez-Valle, M., Vance, M., Moolhuijzen, P. M., Tao, X., and Lew-Tabor, A. E. (2012). Differential recognition by tick-resistant cattle of the recombinantly expressed Rhipicephalus microplus serine protease inhibitor-3 (RMS-3). Ticks Tick Borne Dis. 3, 159-169. doi: 10.1016/j.ttbdis.2012.03.002

Rodriguez-Valle, M., Xu, T., Kurscheid, S., and Lew-Tabor, A. E. (2015). Rhipicephalus microplus serine protease inhibitor family: annotation, expression and functional characterisation assessment. Parasit. Vectors 8:7. doi: 10.1186/s13071-014-0605-4

Salát, J., Paesen, G. C., Rezácová, P., Kotsyfakis, M., Kovárová, Z., Sanda, M., et al. (2010). Crystal structure and functional characterization of an immunomodulatory salivary cystatin from the soft tick Ornithodoros moubata. Biochem. J. 429, 103-112. doi: 10.1042/BJ20100280

Sandhaus, R. A., and Turino, G. (2013). Neutrophil elastase-mediated lung disease. COPD 10 (Suppl. 1), 60-63. doi: 10.3109/15412555.2013.764403

Sa-Nunes, A., Bafica, A., Antonelli, L. R., Choi, E. Y., Francischetti, I. M., Andersen, J. F., et al. (2009). The immunomodulatory action of sialostatin $\mathrm{L}$ on dendritic cells reveals its potential to interfere with autoimmunity. J. Immunol. 182, 7422-7429. doi: 10.4049/jimmunol.0900075

Sasaki, S. D., De Lima, C. A., Lovato, D. V., Juliano, M. A., Torquato, R. J., and Tanaka, A. S. (2008). BmSI-7, a novel subtilisin inhibitor from Boophilus microplus, with activity toward $\operatorname{Pr} 1$ proteases from the fungus Metarhizium anisopliae. Exp. Parasitol. 118, 214-220. doi: 10.1016/j.exppara.2007. 08.003

Scherer, H. U., Dorner, T., and Burmester, G. R. (2010). Patient-tailored therapy in rheumatoid arthritis: an editorial review. Curr. Opin. Rheumatol. 22, 237-245. doi: 10.1097/BOR.0b013e328337b832

Schmitt, E., Germann, T., Goedert, S., Hoehn, P., Huels, C., Koelsch, S., et al. (1994). IL-9 production of naive CD4+ T cells depends on IL-2, is synergistically enhanced by a combination of TGF-beta and IL-4, and is inhibited by IFN-gamma. J. Immunol. 153, 3989-3996.

Schwarz, A., Cabezas-Cruz, A., Kopecky, J., and Valdes, J. J. (2014). Understanding the evolutionary structural variability and target specificity of tick salivary Kunitz peptides using next generation transcriptome data. BMC Evol. Biol. 14:4. doi: 10.1186/1471-2148-14-4

Schwarz, A., Valdes, J. J., and Kotsyfakis, M. (2012). The role of cystatins in tick physiology and blood feeding. Ticks Tick Borne Dis. 3, 117-127. doi: 10.1016/j.ttbdis.2012.03.004

Schwarz, A., Von Reumont, B. M., Erhart, J., Chagas, A. C., Ribeiro, J. M., and Kotsyfakis, M. (2013). De novo Ixodes ricinus salivary gland transcriptome analysis using two next-generation sequencing methodologies. FASEB J. 27, 4745-4756. doi: 10.1096/fj.13-232140

Shapiro, S. D. (2002). Proteinases in chronic obstructive pulmonary disease. Biochem. Soc. Trans. 30, 98-102. doi: 10.1042/bst0300098

Shi, X., Gangadharan, B., Brass, L. F., Ruf, W., and Mueller, B. M. (2004). Protease-activated receptors (PAR1 and PAR2) contribute to tumor cell motility and metastasis. Mol. Cancer Res. 2, 395-402. Available online at: http://mcr. aacrjournals.org/content/2/7/395.article-info

Silverman, G. A., Whisstock, J. C., Bottomley, S. P., Huntington, J. A., Kaiserman, D., Luke, C. J., et al. (2010). Serpins flex their muscle: I. Putting the clamps on proteolysis in diverse biological systems. J. Biol. Chem. 285, 24299-24305. doi: $10.1074 /$ jbc.R110.112771

Stark, K. R., and James, A. A. (1995). A factor Xa-directed anticoagulant from the salivary glands of the yellow fever mosquito Aedes aegypti. Exp. Parasitol. 81, 321-331. doi: 10.1006/expr.1995.1123

Stark, K. R., and James, A. A. (1998). Isolation and characterization of the gene encoding a novel factor Xa-directed anticoagulant from the yellow fever mosquito, Aedes aegypti. J. Biol. Chem. 273, 20802-20809. doi: $10.1074 / j b c .273 .33 .20802$

Steinwede, K., Maus, R., Bohling, J., Voedisch, S., Braun, A., Ochs, M., et al. (2012). Cathepsin G and neutrophil elastase contribute to lung-protective immunity against mycobacterial infections in mice. J. Immunol. 188, 4476-4487. doi: 10.4049/jimmunol.1103346

Stephenson, E., Savvatis, K., Mohiddin, S. A., and Marelli-Berg, F. M. (2016). Tcell immunity in myocardial inflammation: pathogenic role and therapeutic manipulation. Br. J. Pharmacol. doi: 10.1111/bph.13613

Sudhan, D. R., and Siemann, D. W. (2015). Cathepsin L targeting in cancer treatment. Pharmacol. Ther. 155, 105-116. doi: 10.1016/j.pharmthera.2015.08.007

Sugino, M., Imamura, S., Mulenga, A., Nakajima, M., Tsuda, A., Ohashi, K., et al. (2003). A serine proteinase inhibitor (serpin) from ixodid tick Haemaphysalis longicornis; cloning and preliminary assessment of its suitability as a candidate for a tick vaccine. Vaccine 21, 2844-2851. doi: 10.1016/S0264-410X(03) 00167-1

Sun, J., Sukhova, G. K., Wolters, P. J., Yang, M., Kitamoto, S., Libby, P., et al. (2007). Mast cells promote atherosclerosis by releasing proinflammatory cytokines. Nat. Med. 13, 719-724. doi: 10.1038/nm1601

Sun, J., Zhang, J., Lindholt, J. S., Sukhova, G. K., Liu, J., He, A., et al. (2009). Critical role of mast cell chymase in mouse abdominal aortic aneurysm formation. Circulation 120, 973-982. doi: 10.1161/CIRCULATIONAHA.109.849679

Takeda, A., Jimi, T., Wakayama, Y., Misugi, N., Miyake, S., and Kumagai, T. (1992). Demonstration of cathepsins B, H and L in xenografts of normal and Duchenne-muscular-dystrophy muscles transplanted into nude mice. Biochem. J. 288 ( Pt 2), 643-648. doi: 10.1042/bj2880643

Talukdar, S., Oh, D. Y., Bandyopadhyay, G., Li, D., Xu, J., Mcnelis, J., et al. (2012). Neutrophils mediate insulin resistance in mice fed a high-fat diet through secreted elastase. Nat. Med. 18, 1407-1412. doi: 10.1038/nm.2885

Tan, G. J., Peng, Z. K., Lu, J. P., and Tang, F. Q. (2013). Cathepsins mediate tumor metastasis. World J. Biol. Chem. 4, 91-101. doi: 10.4331/wjbc.v4.i4.91

Tang, H. (2009). Regulation and function of the melanization reaction in Drosophila. Fly (Austin) 3, 105-111. doi: 10.4161/fly.3.1.7747

Tirloni, L., Kim, T. K., Coutinho, M. L., Ali, A., Seixas, A., Termignoni, C., et al. (2016). The putative role of Rhipicephalus microplus salivary serpins in the tick-host relationship. Insect. Biochem. Mol. Biol. 71, 12-28. doi: 10.1016/j.ibmb.2016.01.004

Tirloni, L., Reck, J., Terra, R. M., Martins, J. R., Mulenga, A., Sherman, N. E., et al. (2014a). Proteomic analysis of cattle tick Rhipicephalus (Boophilus) microplus 
saliva: a comparison between partially and fully engorged females. PLoS ONE 9:e94831. doi: 10.1371/journal.pone.0094831

Tirloni, L., Seixas, A., Mulenga, A., Vaz Ida, S. Jr., and Termignoni, C. (2014b). A family of serine protease inhibitors (serpins) in the cattle tick Rhipicephalus (Boophilus) microplus. Exp. Parasitol. 137, 25-34. doi: 10.1016/j.exppara.2013.12.001

Toyomane, K., Konnai, S., Niwa, A., Githaka, N., Isezaki, M., Yamada, S., et al. (2016). Identification and the preliminary in vitro characterization of IRIS homologue from salivary glands of Ixodes persulcatus Schulze. Ticks Tick Borne Dis. 7, 119-125. doi: 10.1016/j.ttbdis.2015.09.006

Tumminello, F. M., Leto, G., Pizzolanti, G., Candiloro, V., Crescimanno, M., Crosta, L., et al. (1996). Cathepsin D, B and L circulating levels as prognostic markers of malignant progression. Anticancer Res. 16, 2315-2319.

Turk, V., Stoka, V., Vasiljeva, O., Renko, M., Sun, T., Turk, B., et al. (2012). Cysteine cathepsins: from structure, function and regulation to new frontiers. Biochim. Biophys. Acta 1824, 68-88. doi: 10.1016/j.bbapap.2011. 10.002

Twigg, M. S., Brockbank, S., Lowry, P., Fitzgerald, S. P., Taggart, C., and Weldon, S. (2015). The Role of Serine Proteases and Antiproteases in the Cystic Fibrosis Lung. Mediators Inflamm. 2015:293053. doi: 10.1155/2015/293053

Valdes, J. J., and Moal, I. H. (2014). Prediction of Kunitz ion channel effectors and protease inhibitors from the Ixodes ricinus sialome. Ticks Tick Borne Dis. 5, 947-950. doi: 10.1016/j.ttbdis.2014.07.016

Valenzuela, J. G., Francischetti, I. M., Pham, V. M., Garfield, M. K., Mather, T. N., and Ribeiro, J. M. (2002). Exploring the sialome of the tick Ixodes scapularis. J. Exp. Biol. 205, 2843-2864. Available online at: http://jeb.biologists.org/content/ 205/18/2843.article-info

Wagner, C. J., Schultz, C., and Mall, M. A. (2016). Neutrophil elastase and matrix metalloproteinase 12 in cystic fibrosis lung disease. Mol Cell Pediatr 3:25. doi: 10.1186/s40348-016-0053-7

Wang, Y., Yu, X., Cao, J., Zhou, Y., Gong, H., Zhang, H., et al. (2015a). Characterization of a secreted cystatin from the tick Rhipicephalus haemaphysaloides. Exp. Appl. Acarol. 67, 289-298. doi: 10.1007/s10493-015-9946-8

Wang, Y., Zhou, Y., Gong, H., Cao, J., Zhang, H., Li, X., et al. (2015b). Functional characterization of a cystatin from the tick Rhipicephalus haemaphysaloides. Parasit. Vectors 8:140. doi: 10.1186/s13071-015-0725-5

Whisstock, J. C., Silverman, G. A., Bird, P. I., Bottomley, S. P., Kaiserman, D., Luke, C. J., et al. (2010). Serpins flex their muscle: II. Structural insights into target peptidase recognition, polymerization, and transport functions. J. Biol. Chem. 285, 24307-24312. doi: 10.1074/jbc.R110.141408

Wikel, S. (2013). Ticks and tick-borne pathogens at the cutaneous interface: host defenses, tick countermeasures, and a suitable environment for pathogen establishment. Front. Microbiol. 4:337. doi: 10.3389/fmicb.2013. 00337

Willadsen, P., Bird, P., Cobon, G. S., and Hungerford, J. (1995). Commercialisation of a recombinant vaccine against Boophilus microplus. Parasitology 110 (Suppl.), S43-S50. doi: 10.1017/S0031182000001487

Xu, T., Lew-Tabor, A., and Rodriguez-Valle, M. (2016). Effective inhibition of thrombin by Rhipicephalus microplus serpin-15 (RmS-15) obtained in the yeast Pichia pastoris. Ticks Tick Borne Dis. 7, 180-187. doi: 10.1016/j.ttbdis.2015.09.007

Yamaji, K., Tsuji, N., Miyoshi, T., Hatta, T., Alim, M. A., Anisuzzaman, et al. (2010). Hlcyst-1 and Hlcyst-2 are potential inhibitors of HlCPL-A in the midgut of the ixodid tick Haemaphysalis longicornis. J. Vet. Med. Sci. 72, 599-604. doi: 10.1292/jvms.09-0561

Yamaji, K., Tsuji, N., Miyoshi, T., Islam, M. K., Hatta, T., Alim, M. A., et al. (2009a). Hemoglobinase activity of a cysteine protease from the ixodid tick Haemaphysalis longicornis. Parasitol. Int. 58, 232-237. doi: 10.1016/j.parint.2009.05.003

Yamaji, K., Tsuji, N., Miyoshi, T., Islam, M. K., Hatta, T., Alim, M. A., et al. (2009b). A salivary cystatin, HISC-1, from the ixodid tick Haemaphysalis longicornis play roles in the blood-feeding processes. Parasitol. Res. 106, 61-68. doi: 10.1007/s00436-009-1626-3

Yasuda, Y., Kaleta, J., and Bromme, D. (2005). The role of cathepsins in osteoporosis and arthritis: rationale for the design of new therapeutics. Adv. Drug Deliv. Rev. 57, 973-993. doi: 10.1016/j.addr.2004.12.013

Yu, Y., Cao, J., Zhou, Y., Zhang, H., and Zhou, J. (2013) Isolation and characterization of two novel serpins from the tick Rhipicephalus haemaphysaloides. Ticks Tick Borne Dis. 4, 297-303. doi: 10.1016/j.ttbdis.2013.02.001

Zavasnik-Bergant, T. (2008). Cystatin protease inhibitors and immune functions. Front. Biosci. 13, 4625-4637. doi: 10.2741/3028

Zeng, X. C., Liu, Y., Shi, W., Zhang, L., Luo, X., Nie, Y., et al. (2014). Genome-wide search and comparative genomic analysis of the trypsin inhibitor-like cysteine-rich domain-containing peptides. Peptides 53, 106-114. doi: 10.1016/j.peptides.2013.08.012

Zhang, J., Sun, J., Lindholt, J. S., Sukhova, G. K., Sinnamon, M., Stevens, R. L., et al. (2011). Mast cell tryptase deficiency attenuates mouse abdominal aortic aneurysm formation. Circ. Res. 108, 1316-1327. doi: 10.1161/CIRCRESAHA.111.243758

Zhou, J., Liao, M., Gong, H., Xuan, X., and Fujisaki, K. (2010). Characterization of Hlcyst-3 as a member of cystatins from the tick Haemaphysalis longicornis. Exp. Appl. Acarol. 51, 327-333. doi: 10.1007/s10493-010-9336-1

Zhou, J., Liao, M., Hatta, T., Tanaka, M., Xuan, X., and Fujisaki, K. (2006a). Identification of a follistatin-related protein from the tick Haemaphysalis longicornis and its effect on tick oviposition. Gene 372, 191-198. doi: 10.1016/j.gene.2005.12.020

Zhou, J., Liao, M., Ueda, M., Gong, H., Xuan, X., and Fujisaki, K. (2009). Characterization of an intracellular cystatin homolog from the tick Haemaphysalis longicornis. Vet. Parasitol. 160, 180-183. doi: 10.1016/j.vetpar.2008.10.086

Zhou, J., Ueda, M., Umemiya, R., Battsetseg, B., Boldbaatar, D., Xuan, X., et al. (2006b). A secreted cystatin from the tick Haemaphysalis longicornis and its distinct expression patterns in relation to innate immunity. Insect Biochem. Mol. Biol. 36, 527-535. doi: 10.1016/j.ibmb.2006. 03.003

Conflict of Interest Statement: The authors declare that the research was conducted in the absence of any commercial or financial relationships that could be construed as a potential conflict of interest.

Copyright (c) 2017 Chmelař, Kotál, Langhansová and Kotsyfakis. This is an openaccess article distributed under the terms of the Creative Commons Attribution License (CC BY). The use, distribution or reproduction in other forums is permitted, provided the original author(s) or licensor are credited and that the original publication in this journal is cited, in accordance with accepted academic practice. No use, distribution or reproduction is permitted which does not comply with these terms. 